Eirkhäuser 


\section{Operator Theory: Advances and Applications}

Volume 272

\section{Founded in 1979 by Israel Gohberg}

Series Editors:

Joseph A. Ball (Blacksburg, VA, USA)

Albrecht Böttcher (Chemnitz, Germany)

Harry Dym (Rehovot, Israel)

Heinz Langer (Wien, Austria)

Christiane Tretter (Bern, Switzerland)

\section{Associate Editors:}

Vadim Adamyan (Odessa, Ukraine)

Wolfgang Arendt (Ulm, Germany)

B. Malcolm Brown (Cardiff, UK)

Raul Curto (Iowa, IA, USA)

Kenneth R. Davidson (Waterloo, ON, Canada)

Fritz Gesztesy (Waco, TX, USA)

Pavel Kurasov (Stockholm, Sweden)

Vern Paulsen (Houston, TX, USA)

Mihai Putinar (Santa Barbara, CA, USA)

Ilya Spitkovsky (Abu Dhabi, UAE)

\author{
Honorary and Advisory Editorial Board: \\ Lewis A. Coburn (Buffalo, NY, USA) \\ Ciprian Foias (College Station, TX, USA) \\ J.William Helton (San Diego, CA, USA) \\ Marinus A. Kaashoek (Amsterdam, NL) \\ Thomas Kailath (Stanford, CA, USA) \\ Peter Lancaster (Calgary, Canada) \\ Peter D. Lax (New York, NY, USA) \\ Bernd Silbermann (Chemnitz, Germany) \\ Harold Widom (Santa Cruz, CA, USA)
}

\section{Subseries \\ Linear Operators and Linear Systems}

Subseries editors:

Daniel Alpay (Orange, CA, USA)

Birgit Jacob (Wuppertal, Germany)

André C.M. Ran (Amsterdam, The Netherlands)

\section{Subseries}

\section{Advances in Partial Differential Equations}

Subseries editors:

Bert-Wolfgang Schulze (Potsdam, Germany)

Michael Demuth (Clausthal, Germany)

Jerome A. Goldstein (Memphis, TN, USA)

Nobuyuki Tose (Yokohama, Japan)

Ingo Witt (Göttingen, Germany)

More information about this series at http://www.springer.com/series/4850 
Vladimir Bolotnikov • Sanne ter Horst • André C.M. Ran Victor Vinnikov

Editors

\section{Interpolation and Realization Theory with Applications to Control Theory}

In Honor of Joe Ball 


\section{Editors}

Vladimir Bolotnikov

Department of Mathematics

The College of William and Mary

Williamsburg, VA, USA

André C.M. Ran

Department of Mathematics

Faculty of Science, VU Amsterdam

Amsterdam, The Netherlands
Sanne ter Horst

Department of Mathematics

Unit for BMI, North-West University

Potchefstroom, South Africa

Victor Vinnikov

Department of Mathematics

Ben Gurion University of the Negev

Be'er-Sheva, Israel

ISSN 0255-0156

Operator Theory: Advances and Applications

ISSN 2296-4878 (electronic)

ISBN 978-3-030-11613-2

ISBN 978-3-030-11614-9 (eBook)

https://doi.org/10.1007/978-3-030-11614-9

Mathematics Subject Classification (2010): 47-XX

(C) Springer Nature Switzerland AG 2019

This work is subject to copyright. All rights are reserved by the Publisher, whether the whole or part of the material is concerned, specifically the rights of translation, reprinting, reuse of illustrations, recitation, broadcasting, reproduction on microfilms or in any other physical way, and transmission or information storage and retrieval, electronic adaptation, computer software, or by similar or dissimilar methodology now known or hereafter developed.

The use of general descriptive names, registered names, trademarks, service marks, etc. in this publication does not imply, even in the absence of a specific statement, that such names are exempt from the relevant protective laws and regulations and therefore free for general use.

The publisher, the authors and the editors are safe to assume that the advice and information in this book are believed to be true and accurate at the date of publication. Neither the publisher nor the authors or the editors give a warranty, expressed or implied, with respect to the material contained herein or for any errors or omissions that may have been made. The publisher remains neutral with regard to jurisdictional claims in published maps and institutional affiliations.

This book is published under the imprint Birkhäuser, www.birkhauser-science.com by the registered company Springer Nature Switzerland AG.

The registered company address is: Gewerbestrasse 11, 6330 Cham, Switzerland 


\section{Contents}

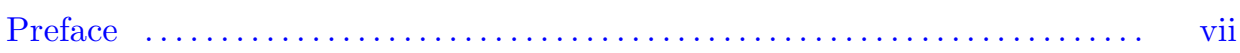

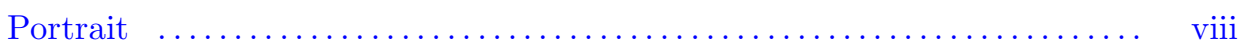

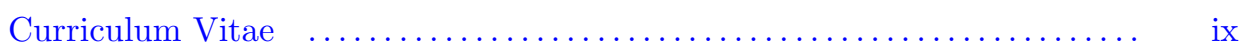

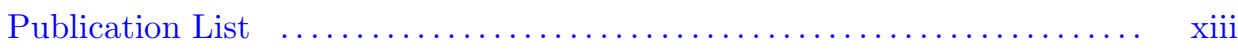

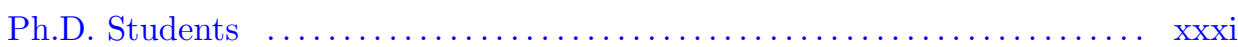

Personal Reminiscences $\ldots \ldots \ldots \ldots \ldots \ldots \ldots \ldots \ldots \ldots \ldots \ldots \ldots \ldots \ldots \ldots \ldots \ldots \ldots$ xxiii

Y. Arlinskiu and S. Hassi

Holomorphic Operator-valued Functions Generated

by Passive Selfadjoint Systems $\ldots \ldots \ldots \ldots \ldots \ldots \ldots \ldots \ldots \ldots \ldots \ldots$

M. Augat, J.W. Helton, I. Klep and S. McCullough

Free Bianalytic Maps between Spectrahedra and

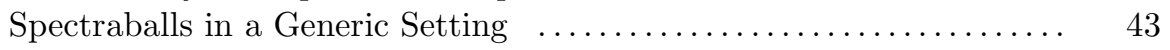

S. Balasubramanian, S. McCullough and U. Wijesooriya

Szegö and Widom Theorems for the Neil Algebra $\ldots . \ldots \ldots \ldots \ldots . . . .61$

N. Cohen

Block Triangular Matrices in Banach Space:

Minimal Completions and Factorability $\ldots \ldots \ldots \ldots \ldots \ldots \ldots \ldots . \ldots \ldots$

Q. Fang

Multipliers of Drury-Arveson Space: A Survey $\ldots . \ldots \ldots \ldots \ldots \ldots . . \ldots 9$

S. Gorai and J. Sarkar

Contractively Embedded Invariant Subspaces $\ldots \ldots \ldots \ldots \ldots \ldots \ldots . \ldots 117$

G.J. Groenewald, S. ter Horst, J. Jaftha and A.C.M. Ran

A Toeplitz-like Operator with Rational Symbol

Having Poles on the Unit Circle II: The Spectrum 
S. ter Horst, M.A. Kaashoek and F. van Schagen

The Twofold Ellis-Gohberg Inverse Problem in an Abstract

Setting and Applications

A. Kheifets

Abstract Interpolation Problem and Some Applications.

II: Coefficient Matrices.

Z.A. Lykova, N.J. Young and A.E. Ajibo

Analytic Interpolation into the Tetrablock and

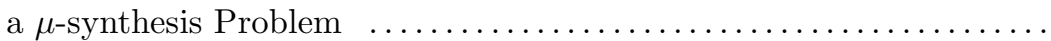

R.T.W. Martin and A. Ramanantoanina

A Gleason Solution Model for Row Contractions ............... 249

M.-J.Y. Ou and H.J. Woerdeman

On the Augmented Biot-JKD Equations with Pole-Residue

Representation of the Dynamic Tortuosity

A. van der Schaft

The Flow Equations of Resistive

Electrical Networks

A. Tannenbaum, T.T. Georgiou, J.O. Deasy and L. Norton

Control and the Analysis of Cancer Growth Models 


\section{Preface}

This volume in the Operator Theory: Advances and Application series is devoted to Joseph A. (Joe) Ball's four and a half decade long mathematical career and in celebration of his seventieth birthday on June 4, 2017. His exemplary dedication to mathematics was also recognized in a special session and dinner dedicated to Joe's Birthday at the 34th Southeastern Analysis Meeting (SEAM 2018), at Georgia Tech, organized by Quanlei Fang, and his election to the class of 2019 fellows of the American Mathematical Society.

Joe started out working on model theory and related topics for non-contractions and operators on multiply-connected domains. After he got into contact with Bill Helton from UCSD in the 1970s, more applied operator theory themes appeared in his work, involving factorization and interpolation for operator-valued functions, with extensive applications in system and control theory. This culminated in his 1990 monograph Interpolation of rational matrix functions (OT 45) written jointly with Israel Gohberg and Leiba Rodman. He has since worked on nonlinear control, time-varying systems and more recently on multidimensional systems and noncommutative $H^{\infty}$-theory on the unit ball and polydisk, and more general domains, and these are only the main themes in his vast oeuvre. It came as a shock to many when Joe announced he was going to retire in 2016, but he quickly reassured us that it is only retirement from regular teaching. And, indeed, his productivity has not suffered under this, to the contrary. In total Joe published more than 200 research papers, over 40 proceedings papers and supervised fourteen Ph.D. students. Further details can be found in Joe's curriculum vitae, publication list and list of Ph.D. students included elsewhere in this volume. There is also a chapter that includes personal reminiscences by some of his collaborators, colleagues and friends.

However, the main bulk of this volume is reserved for fourteen research papers on topics in operator theory and it's application, by mathematicians, many of whom collaborated with Joe or were in some other way influenced by his work. We thank all the authors who contributed to this volume for their efforts as well as the referees who in many cases helped to significantly improve the manuscripts.

We dedicate this volume to Joe, with gratitude for the many things we learned from him, and we wish him many fruitful years to go.

The editors

Vladimir Bolotnikov, Sanne ter Horst, André Ran, Victor Vinnikov 


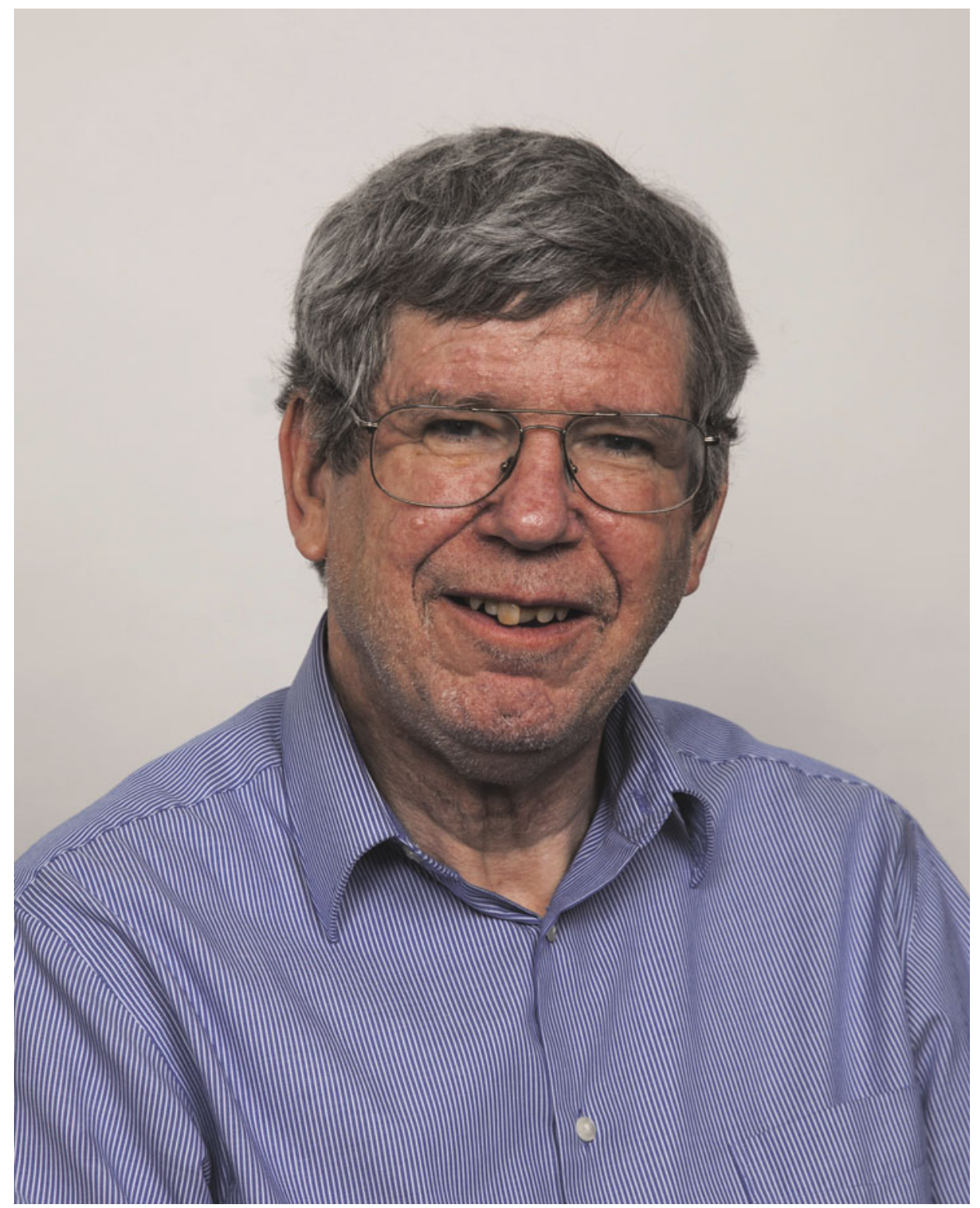

Joseph A. Ball 


\section{Curriculum Vitae of Joseph A. Ball}

\section{Personal data}

Name: Joseph (Joe) A. Ball

Date of birth: June 4, 1947

Place of birth: Washington, D.C., USA

\section{Research field and research interests}

Operator theory, engineering systems theory, robust control theory, complex analysis.

\section{Education}

1969 B.S. in Mathematics, Georgetown University, Washington, D.C.

1970 M.S. in Mathematics, University of Virginia, Charlottesville, VA.

1973 Ph.D. in Mathematics, University of Virginia, Charlottesville, VA.

Dissertation: "Unitary Perturbations of Contraction Operators" Advisor: Marvin Rosenblum

\section{Academic appointments}

- Assistant Professor, Department of Mathematics, Virginia Tech, Blacksburg, VA, September 1973-August 1978.

- Associate Professor, Department of Mathematics, Virginia Tech, Blacksburg, VA, September 1978-August 1982.

- Professor, Department of Mathematics, Virginia Tech, Blacksburg, VA, September 1982-June 2016.

- Professor Emeritus, Department of Mathematics, Virginia Tech, Blacksburg, VA, September 2016-present.

\section{Visiting professorships}

- Mathematician, Dahlgren U.S. Navy Weapons Research Lab, Summer 1975.

- Visiting Assistant Professor, Department of Mathematics, University of California at San Diego, La Jolla, CA, January-June 1978 and September 1979June 1980.

- Visiting Professor, Weizmann Institute of Science, Rehovot, Israel, JanuaryJune 1983. 
- Visiting Professor, University of California at San Diego, La Jolla, CA, March-June 1987 and March-June 1991.

- Full Member of the Mathematical Sciences Research Institute "Holomorphic Spaces" program, Berkeley, California, September-December 1995.

\section{Research outputs}

- Over 140 papers in refereed professional journals, 60 refereed book chapters and 40 conference proceedings papers.

- two research monographs and two AMS memoirs.

- Co-editor of eight conference proceedings and special book volumes.

- Fourteen Ph.D. student dissertations directed.

- Seven M.S. students supervised.

\section{Lectures at professional meetings}

Over 165 lectures at national and international workshops and conferences, including more than

- 25 at American Mathematical Society Meetings;

- 25 at International Workshops on Operator Theory and it's Applications (IWOTA);

- 19 at International Symposia on Mathematical Theory of Network and Systems (MTNS);

- 12 at South East Analysis Meetings (SEAM);

- and 10 at conferences of the Society for Industrial and Applied Mathematics (SIAM).

Plenary and semi-plenary lectures:

- IWOTA 2005, Storrs, CT, USA. Title: Multidimensional system theory, LaxPhillips scattering and multivariable operator theory: the polydisk setting.

- IWOTA 2006, Seoul, South Korea. Title: Transfer function realization and Nevanlinna-Pick interpolation for general classes of nonselfadjoint operator algebras.

- IWOTA 2007, Potchefstroom, South Africa. Title: Multivariable generalizations of the Schur class, completely positive kernels and multidimensionla linear systems.

- IWOTA 2009, Guanajuato, Mexico. Title Discrete-time overdetermined (Livšic) linear systems, algebraic curves, and meromorphic bundle maps: examples and applications.

- IWOTA 2010 Berlin, Germany. Title: Canonical model theory for Hilbert space row contractions.

- MTNS 2010, Budapest, Hungary. Title: Robust Control, Multidimensional Systems and Multivariable Function Theory: Commutative and Noncommutative Settings.

- IWOTA 2011, Seville, Spain. Title: The intertwining of function theory and systems engineering. 
- IWOTA 2012, Sydney Australia. Title: Input/state/output linear systems and function theory: the Bergman space setting.

- IWOTA 2013, Bangalore, India. Title: Transfer function realization and zero/ pole structure for multivariable rational matrix functions: the direct analysis.

- IWOTA 2015, Tbilisi, Georgia. Title: Multivariable Nevanlinna-Pick interpolation: the free noncommutative setting.

\section{Editorial work}

Member of the Editorial Board of

- Integral Equations and Operator Theory, 1984-present.

- Systems \& Control Letters, 1987-1992.

- Journal of the Mathematics of Systems, Estimation, and Control, 1990-1996.

- Journal of Mathematical Analysis and Applications, 1994-present.

- Proceedings of the American Mathematical Society, 1999-2007.

- Complex Analysis and Operator Theory, 2006-present.

- The Birkhäuser book series Operator Theory: Advances and Applications, 2009-present.

- Banach Journal of Mathematical Analysis, 2010-present.

- Multidimensional Signals \& Systems, 2011-present.

\section{Refereeing and reviewing}

- Refereed research papers for over 70 journals.

- Reviewer for Zentralblatt für Mathematik und ihre Grenzgebiete (over 110 reviews) and Mathematical Reviews (over 350 reviews).

- Served on numerous graduate student advisor committees.

- Served on several undergraduate honor student oral exam committees.

- Reviewer of grant proposals and prospective research monographs.

\section{Conference organization}

- Member of Steering Committee for the International Symposia on Mathematical Theory of Networks and Systems (MTNS), 2002-present.

- Member of Steering Committee for the International Workshop on Operator Theory and Applications (IWOTA), 2002-present.

- Member of IWOTA Presidium, 2009-present.

- Member of local organizing committee for IWOTA 2002, Virginia Tech, Blacksburg, VA, USA, August 6-9, 2002.

- Member of scientific committee for IWOTA 2003, Cagliari, Sardinia, Italy, June 24-27, 2003.

- Member of local organizing committee for IWOTA 2008, College of William \& Mary, Williamsburg, VA, USA, July 22-26, 2008.

- Member of local organizing committee for MTNS 2008, Virginia Tech, Blacksburg, VA, USA, July 28-August 1, 2008.

- Member of local organizing committee for Southeast Analysis Meeting (SEAM) 2013, Virginia Tech, Blacksburg, VA, USA, March 15-16, 2013. 
- Co-organizer of special sessions at numerous AMS, IWOTA and MTNS conferences.

\section{Research grants and awards}

- Supported participant at the NSF Operator Theory Institute at the University of New Hampshire, 1976.

- US National Science Foundation research grant, 1977-1987 (with R.F. Olin and J.E. Thomson), 1978-1998, 2000-2003.

- Co-principal investigator (with P. Kachroo of Center for Transportation Research, Virginia Tech) for Federal Highway Administration Grant DTFG6193-X-00017-002, 1996-1997.

- Alumni Award for Research Excellence, Virginia Tech, 1997.

- US-Israeli Binational Science Foundation grant, 2000-2007 (with D. Alpay, C. Sadosky and V. Vinnikov), 2011-2014 (with D. Kaliuzhnyi-Verbovetskyi and Victor Vinnikov).

- Co-Principal investigator (with M. Klaus, L. Rodman and J.W. Helton) for NSF Grant \#DMS-0126746 "Thirteenth International Workshop on Operator Theory and Applications", 2002-2003.

- Co-Principal investigator (with M. Klaus) for NSF Grant DMS-1266053 "Conference/Workshop: Southeastern Analysis Meeting SEAM 2013", 20122013.

- Fellow of the American Mathematical Society, class of 2019.

\section{Memberships of professional organizations}

- American Mathematical Society.

- Society of Industrial and Applied Mathematicians. 


\section{Publication List of J.A. Ball}

\section{Dissertation}

[1] J.A. Ball, Unitary perturbations of contractions, ProQuest LLC, Ann Arbor, MI, 1973. Thesis (Ph.D.) - University of Virginia.

\section{Research monographs}

[1] J.W. Helton with the assistance of J.A. Ball, C.R. Johnson and J.N. Palmer, Operator theory, analytic functions, matrices, and electrical engineering, CBMS Regional Conference Series in Mathematics, vol. 68, Published for the Conference Board of the Mathematical Sciences, Washington, DC; by the American Mathematical Society, Providence, RI, 1987.

[2] J.A. Ball, I. Gohberg, and L. Rodman, Interpolation of rational matrix functions, Operator Theory: Advances and Applications, vol. 45, Birkhäuser Verlag, Basel, 1990.

\section{Papers in professional journals}

[1] J.A. Ball, Factorization and invariant subspaces for noncontractions, Bull. Amer. Math. Soc. 80 (1974), 896-900.

[2] J.A. Ball, Hardy space expectation operators and reducing subspaces, Proc. Amer. Math. Soc. 47 (1975), 351-357.

[3] J.A. Ball, Models for noncontractions, J. Math. Anal. Appl. 52 (1975), no. 2, 235254.

[4] J.A. Ball, A norm estimate for an integral operator, Transport Theory Statist. Phys. 4 (1975), no. 2, 67-69.

[5] J.A. Ball and W. Greenberg, A Pontrjagin space analysis of the supercritical transport equation, Transport Theory Statist. Phys. 4 (1975), no. 4, 143-154.

[6] J.A. Ball and A. Lubin, On a class of contractive perturbations of restricted shifts, Pacific J. Math. 63 (1976), no. 2, 309-323.

[7] M.B. Abrahamse and J.A. Ball, Analytic Toeplitz operators with automorphic symbol. II, Proc. Amer. Math. Soc. 59 (1976), no. 2, 323-328.

[8] J.A. Ball, Rota's theorem for general functional Hilbert spaces, Proc. Amer. Math. Soc. 64 (1977), no. 1, 55-61. 
[9] J.A. Ball, R.F. Olin, and J.E. Thomson, Weakly closed algebras of subnormal operators, Illinois J. Math. 22 (1978), no. 2, 315-326.

[10] J.A. Ball, Factorization and model theory for contraction operators with unitary part, Mem. Amer. Math. Soc. 13 (1978), no. 198, iv+68.

[11] J.A. Ball, Operators of class $C_{00}$ over multiply-connected domains, Michigan Math. J. 25 (1978), no. 2, 183-196.

[12] J.A. Ball, A lifting theorem for operator models of finite rank on multiply-connected domains, J. Operator Theory 1 (1979), no. 1, 3-25.

[13] J.A. Ball, Operator extremal problems, expectation operators and applications to operators on multiply connected domains, J. Operator Theory 1 (1979), no. 2, 153175.

[14] J.A. Ball and J.W. Helton, Nonnormal dilations, disconjugacy and constrained spectral factorization, Integral Equations Operator Theory 3 (1980), no. 2, 216-309.

[15] J.A. Ball, Interpolation problems and Toeplitz operators on multiply connected domains, Integral Equations Operator Theory 4 (1981), no. 2, 172-184.

[16] J.A. Ball and J.W. Helton, Subinvariants for analytic mappings on matrix balls, Analysis 1 (1981), no. 3, 217-226.

[17] J.W. Helton and J.A. Ball, The cascade decompositions of a given system vs. the linear fractional decompositions of its transfer function, Integral Equations Operator Theory 5 (1982), no. 3, 341-385.

[18] J.A. Ball and J.W. Helton, Lie groups over the field of rational functions, signed spectral factorization, signed interpolation, and amplifier design, J. Operator Theory 8 (1982), no. 1, 19-64.

[19] J.A. Ball and J.W. Helton, Factorization results related to shifts in an indefinite metric, Integral Equations Operator Theory 5 (1982), no. 5, 632-658.

[20] J.A. Ball and J.W. Helton, A Beurling-Lax theorem for the Lie group $\mathrm{U}(m, n)$ which contains most classical interpolation theory, J. Operator Theory 9 (1983), no. $1,107-142$.

[21] J.A. Ball, Interpolation problems of Pick-Nevanlinna and Loewner types for meromorphic matrix functions, Integral Equations Operator Theory 6 (1983), no. 6, 804-840.

[22] J.A. Ball and J.W. Helton, Beurling-Lax representations using classical Lie groups with many applications. II. $\mathrm{GL}(n, \mathbf{C})$ and Wiener-Hopf factorization, Integral Equations Operator Theory 7 (1984), no. 3, 291-309.

[23] J.A. Ball and I. Gohberg, A commutant lifting theorem for triangular matrices with diverse applications, Integral Equations Operator Theory 8 (1985), no. 2, 205-267.

[24] J.A. Ball and J.W. Helton, Beurling-Lax representations using classical Lie groups with many applications. III. Groups preserving two bilinear forms, Amer. J. Math. 108 (1986), no. 1, 95-174 (1986).

[25] J.A. Ball and I. Gohberg, Shift invariant subspaces, factorization, and interpolation for matrices. I. The canonical case, Linear Algebra Appl. 74 (1986), 87-150.

[26] J.A. Ball and J.W. Helton, Interpolation problems of Pick-Nevanlinna and Loewner types for meromorphic matrix functions: parametrization of the set of all solutions, Integral Equations Operator Theory 9 (1986), no. 2, 155-203. 
[27] J.A. Ball and J.W. Helton, Beurling-Lax representations using classical Lie groups with many applications. IV. $\mathrm{GL}(n, \mathbf{R}), \mathrm{U}^{*}(2 n), \mathrm{SL}(n, \mathbf{C})$, and a solvable group, J. Funct. Anal. 69 (1986), no. 2, 178-206.

[28] J.A. Ball and I. Gohberg, Pairs of shift invariant subspaces of matrices and noncanonical factorization, Linear and Multilinear Algebra 20 (1986), no. 1, 27-61.

[29] J.A. Ball and T.L. Kriete III, Operator-valued Nevanlinna-Pick kernels and the functional models for contraction operators, Integral Equations Operator Theory 10 (1987), no. 1, 17-61.

[30] J.A. Ball and A.C.M. Ran, Global inverse spectral problems for rational matrix functions, Linear Algebra Appl. 86 (1987), 237-282.

[31] J.A. Ball and A.C.M. Ran, Optimal Hankel norm model reductions and WienerHopf factorization. I. The canonical case, SIAM J. Control Optim. 25 (1987), no. 2, 362-382.

[32] J.A. Ball, I. Gohberg, and L. Rodman, Minimal factorization of meromorphic matrix functions in terms of local data, Integral Equations Operator Theory 10 (1987), no. 3, 309-348.

[33] J.A. Ball and A.C.M. Ran, Local inverse spectral problems for rational matrix functions, Integral Equations Operator Theory 10 (1987), no. 3, 349-415.

[34] J.A. Ball and A.C.M. Ran, Optimal Hankel norm model reductions and WienerHopf factorization. II. The noncanonical case, Integral Equations Operator Theory 10 (1987), no. 3, 416-436.

[35] J.A. Ball, C. Foiaş, J.W. Helton, and A. Tannenbaum, On a local nonlinear commutant lifting theorem, Indiana Univ. Math. J. 36 (1987), no. 3, 693-709.

[36] J.A. Ball and A.C.M. Ran, Hankel norm approximation of real symmetric rational matrix functions, Systems Control Lett. 9 (1987), no. 2, 105-115.

[37] J.A. Ball and N. Cohen, Sensitivity minimization in an $H^{\infty}$ norm: parametrization of all suboptimal solutions, Internat. J. Control 46 (1987), no. 3, 785-816.

[38] J.A. Ball, J.W. Helton, and C.H. Sung, Nonlinear solutions of Nevanlinna-Pick interpolation problems, Michigan Math. J. 34 (1987), no. 3, 375-389.

[39] J.A. Ball and J.W. Helton, Shift invariant manifolds and nonlinear analytic function theory, Integral Equations Operator Theory 11 (1988), no. 5, 615-725.

[40] J.A. Ball, C. Foiaş, J.W. Helton, and A. Tannenbaum, A Poincaré-Dulac approach to a nonlinear Beurling-Lax-Halmos theorem, J. Math. Anal. Appl. 139 (1989), no. 2, 496-514.

[41] D.W. Luse and J.A. Ball, Frequency-scale decomposition of $H^{\infty}$-disk problems, SIAM J. Control Optim. 27 (1989), no. 4, 814-835.

[42] J.A. Ball and J.W. Helton, Interconnection of nonlinear causal systems, IEEE Trans. Automat. Control 34 (1989), no. 11, 1132-1140.

[43] J.A. Ball and K.F. Clancey, An elementary description of partial indices of rational matrix functions, Integral Equations Operator Theory 13 (1990), no. 3, 316-322.

[44] J.A. Ball, I. Gohberg, L. Rodman, and T. Shalom, On the eigenvalues of matrices with given upper triangular part, Integral Equations Operator Theory 13 (1990), no. 4, 488-497. 
[45] J.A. Ball, I. Gohberg, and L. Rodman, Simultaneous residue interpolation problems for rational matrix functions, Integral Equations Operator Theory 13 (1990), no. 5, 611-637.

[46] J.A. Ball and M. Rakowski, Minimal McMillan degree rational matrix functions with prescribed local zero-pole structure, Linear Algebra Appl. 137/138 (1990), 325349.

[47] A.C. Antoulas, J.A. Ball, J. Kang, and J.C. Willems, On the solution of the minimal rational interpolation problem, Linear Algebra Appl. 137/138 (1990), 511-573.

[48] J.A. Ball, I. Gohberg, and L. Rodman, Common minimal multiples and divisors for rational matrix functions, Linear Algebra Appl. 137/138 (1990), 621-662.

[49] J.A. Ball and J. Kang, Matrix polynomial solutions of tangential Lagrange-Sylvester interpolation conditions of low McMillan degree, Linear Algebra Appl. 137/138 (1990), 699-746.

[50] J.A. Ball and B. Taub, Factoring spectral matrices in linear-quadratic models, Econom. Lett. 35 (1991), no. 1, 39-44.

[51] J.A. Ball, N. Cohen, and L. Rodman, Zero data and interpolation problems for rectangular matrix polynomials, Linear and Multilinear Algebra 29 (1991), no. 1, $53-78$.

[52] J.A. Ball, J.W. Helton, and M. Verma, A factorization principle for stabilization of linear control systems, International Journal of Robust and Nonlinear Control 1 (1991), 229-294.

[53] J.A. Ball and M. Rakowski, Null-pole subspaces of nonregular rational matrix functions, Linear Algebra Appl. 159 (1991), 81-120.

[54] J.A. Ball, I. Gohberg, and L. Rodman, Boundary Nevanlinna-Pick interpolation for rational matrix functions, J. Math. Systems Estim. Control 1 (1991), no. 2, 131-164.

[55] J.A. Ball and T.R. Fanney, Uniform limits of sequences of polynomials and their derivatives, Proc. Amer. Math. Soc. 114 (1992), no. 3, 749-755.

[56] J.A. Ball and J.W. Helton, Inner-outer factorization of nonlinear operators, J. Funct. Anal. 104 (1992), no. 2, 363-413.

[57] D. Alpay, J.A. Ball, I. Gohberg, and L. Rodman, State space theory of automorphisms of rational matrix functions, Integral Equations Operator Theory 15 (1992), no. 3, 349-377.

[58] J.A. Ball and J.W. Helton, Nonlinear $H^{\infty}$ control theory for stable plants, Math. Control Signals Systems 5 (1992), no. 3, 233-261.

[59] J.A. Ball and E.A. Jonckheere, The four-block Adamjan-Arov-Krĕ̌n problem, J. Math. Anal. Appl. 170 (1992), no. 2, 322-342.

[60] J.A. Ball, J. Kim, L. Rodman, and M. Verma, Minimal-degree coprime factorizations of rational matrix functions, Linear Algebra Appl. 186 (1993), 117-164.

[61] J.A. Ball, J.W. Helton, and M.L. Walker, $H^{\infty}$ control for nonlinear systems with output feedback, IEEE Trans. Automat. Control 38 (1993), no. 4, 546-559.

[62] J.A. Ball, I. Gohberg, and L. Rodman, The structure of flat gain rational matrices that satisfy two-sided interpolation requirements, Systems Control Lett. 20 (1993), no. $6,401-412$. 
[63] J.A. Ball and K.F. Clancey, Interpolation with meromorphic matrix functions, Proc. Amer. Math. Soc. 121 (1994), no. 2, 491-496.

[64] J.A. Ball and J. Kim, Stability and McMillan degree for rational matrix interpolants, Linear Algebra Appl. 196 (1994), 207-232.

[65] J.A. Ball, M.A. Kaashoek, G. Groenewald, and J. Kim, Column reduced rational matrix functions with given null-pole data in the complex plane, Linear Algebra Appl. 203/204 (1994), 67-110.

[66] J.A. Ball, M. Rakowski, and B.F. Wyman, Coupling operators, Wedderburn-Forney spaces, and generalized inverses, Linear Algebra Appl. 203/204 (1994), 111-138.

[67] D. Alpay, J.A. Ball, I. Gohberg, and L. Rodman, J-unitary preserving automorphisms of rational matrix functions: state space theory, interpolation, and factorization, Linear Algebra Appl. 197/198 (1994), 531-566. Second Conference of the International Linear Algebra Society (ILAS) (Lisbon, 1992).

[68] D. Alpay, J.A. Ball, I. Gohberg, and L. Rodman, The two-sided residue interpolation in the Stieltjes class for matrix functions, Linear Algebra Appl. 208/209 (1994), 485-521.

[69] J.A. Ball, I. Gohberg, and M.A. Kaashoek, Bitangential interpolation for inputoutput maps of time-varying systems: the continuous time case, Integral Equations Operator Theory 20 (1994), no. 1, 1-43.

[70] J.A. Ball and M. Verma, Factorization and feedback stabilization for nonlinear systems, Systems Control Lett. 23 (1994), no. 3, 187-196.

[71] J.A. Ball and M. Rakowski, Interpolation by rational matrix functions and stability of feedback systems: the 2-block case, J. Math. Systems Estim. Control 4 (1994), no. 3, 261-318.

[72] J.A. Ball, I. Gohberg, and M. Rakowski, Reconstruction of a rational nonsquare matrix function from local data, Integral Equations Operator Theory 20 (1994), no. 3, 249-305.

[73] J.A. Ball, I. Gohberg, and M.A. Kaashoek, Two-sided Nudel'man interpolation for input-output operators of discrete time-varying systems, Integral Equations Operator Theory 21 (1995), no. 2, 174-211.

[74] J.A. Ball and T.R. Fanney, Pure sub-Jordan operators and simultaneous approximation by a polynomial and its derivative, J. Operator Theory 33 (1995), no. 1, 43-78.

[75] J.A. Ball, I. Gohberg, and M.A. Kaashoek, A frequency response function for linear, time-varying systems, Math. Control Signals Systems 8 (1995), no. 4, 334-351.

[76] J.A. Ball and A.J. van der Schaft, J-inner-outer factorization, J-spectral factorization, and robust control for nonlinear systems, IEEE Trans. Automat. Control 41 (1996), no. 3, 379-392.

[77] J.A. Ball and K.F. Clancey, Reproducing kernels for Hardy spaces on multiply connected domains, Integral Equations Operator Theory 25 (1996), no. 1, 35-57.

[78] J.A. Ball and J. Kim, Bitangential interpolation problems for symmetric rational matrix functions, Linear Algebra Appl. 241/243 (1996), 35-57.

[79] J.A. Ball, Commutant lifting and interpolation: the time-varying case, Integral Equations Operator Theory 25 (1996), no. 4, 377-405. 
[80] J.A. Ball and V. Vinnikov, Zero-pole interpolation for meromorphic matrix functions on an algebraic curve and transfer functions of $2 D$ systems, Acta Appl. Math. 45 (1996), no. 3, 239-316.

[81] J.A. Ball and J.W. Helton, Viscosity solutions of Hamilton-Jacobi equations arising in nonlinear $H_{\infty}$-control, J. Math. Systems Estim. Control 6 (1996), no. 1, 22.

[82] J.A. Ball, I. Gohberg, and M.A. Kaashoek, Nudelman interpolation and the band method, Integral Equations Operator Theory 27 (1997), no. 3, 253-284.

[83] J.A. Ball and T.T. Trent, Unitary colligations, reproducing kernel Hilbert spaces, and Nevanlinna-Pick interpolation in several variables, J. Funct. Anal. 157 (1998), no. $1,1-61$.

[84] J.A. Ball, Yu.I. Karlovich, L. Rodman, and I.M. Spitkovsky, Sarason interpolation and Toeplitz corona theorem for almost periodic matrix functions, Integral Equations Operator Theory 32 (1998), no. 3, 243-281.

[85] J.A. Ball, P. Kachroo, and A.J. Krener, $H_{\infty}$ tracking control for a class of nonlinear systems, IEEE Trans. Automat. Control 44 (1999), no. 6, 1202-1206.

[86] J.A. Ball and V. Vinnikov, Zero-pole interpolation for matrix meromorphic functions on a compact Riemann surface and a matrix Fay trisecant identity, Amer. J. Math. 121 (1999), no. 4, 841-888.

[87] J.A. Ball, W.S. Li, D. Timotin, and T.T. Trent, A commutant lifting theorem on the polydisc, Indiana Univ. Math. J. 48 (1999), no. 2, 653-675.

[88] J.A. Ball, M.V. Day, and P. Kachroo, Robust feedback control of a single server queueing system, Math. Control Signals Systems 12 (1999), no. 4, 307-345.

[89] J.A. Ball, M.V. Day, T. Yu, and P. Kachroo, Robust $L_{2}$-gain control for nonlinear systems with projection dynamics and input constraints: an example from traffic control, Automatica J. IFAC 35 (1999), no. 3, 429-444.

[90] J.A. Ball and J. Chudoung, Comparison theorems for viscosity solutions of a system of quasivariational inequalities with application to optimal control with switching costs, J. Math. Anal. Appl. 251 (2000), no. 1, 40-64.

[91] D. Alpay, J.A. Ball, and V. Bolotnikov, On the bitangential interpolation problem for contractive valued functions in the polydisk, J. Operator Theory 44 (2000), no. 2, 277-301.

[92] D. Alpay, J.A. Ball, and Y. Peretz, System theory, operator models and scattering: the time-varying case, J. Operator Theory 47 (2002), no. 2, 245-286.

[93] J.A. Ball and V. Bolotnikov, On a bitangential interpolation problem for contractive-valued functions on the unit ball, Linear Algebra Appl. 353 (2002), 107-147.

[94] J.A. Ball, J. Chudoung, and M.V. Day, Robust optimal stopping-time control for nonlinear systems, Appl. Math. Optim. 46 (2002), no. 1, 1-29.

[95] J.A. Ball and V. Bolotnikov, A tangential interpolation problem on the distinguished boundary of the polydisk for the Schur-Agler class, J. Math. Anal. Appl. 273 (2002), no. $2,328-348$.

[96] J.A. Ball, J. Chudoung, and M.V. Day, Robust optimal switching control for nonlinear systems, SIAM J. Control Optim. 41 (2002), no. 3, 900-931. 
[97] J.A. Ball and V. Bolotnikov, A bitangential interpolation problem on the closed unit ball for multipliers of the Arveson space, Integral Equations Operator Theory 46 (2003), no. 2, 125-164.

[98] J.A. Ball and M.A. Petersen, Non-linear minimal square spectral factorization, Internat. J. Control 76 (2003), no. 12, 1233-1247.

[99] J.A. Ball and T. Malakorn, Multidimensional linear feedback control systems and interpolation problems for multivariable holomorphic functions, Multidimens. Systems Signal Process. 15 (2004), no. 1, 7-36.

[100] J.A. Ball, M.A. Petersen, and A. van der Schaft, Inner-outer factorization for nonlinear noninvertible systems, IEEE Trans. Automat. Control 49 (2004), no. 4, 483492.

[101] J.A. Ball and V. Bolotnikov, Realization and interpolation for Schur-Agler-class functions on domains with matrix polynomial defining function in $\mathbb{C}^{n}$, J. Funct. Anal. 213 (2004), no. 1, 45-87.

[102] J.A. Ball, C. Sadosky, and V. Vinnikov, Conservative linear systems, unitary colligations and Lax-Phillips scattering: multidimensional generalizations, Internat. J. Control 77 (2004), no. 9, 802-811.

[103] J.A. Ball, C. Sadosky, and V. Vinnikov, Conservative input-state-output systems with evolution on a multidimensional integer lattice, Multidimens. Syst. Signal Process. 16 (2005), no. 2, 133-198.

[104] J.A. Ball and V. Bolotnikov, Nevanlinna-Pick interpolation for Schur-Agler class functions on domains with matrix polynomial defining function in $\mathbb{C}^{n}$, New York J. Math. 11 (2005), 247-290.

[105] J.A. Ball and V. Vinnikov, Lax-Phillips scattering and conservative linear systems: a Cuntz-algebra multidimensional setting, Mem. Amer. Math. Soc. 178 (2005), no. 837 , iv +101 .

[106] J.A. Ball, K.M. Mikkola, and A.J. Sasane, State-space formulas for the NehariTakagi problem for nonexponentially stable infinite-dimensional systems, SIAM J. Control Optim. 44 (2005), no. 2, 531-563.

[107] J.A. Ball, G. Groenewald, and T. Malakorn, Structured noncommutative multidimensional linear systems, SIAM J. Control Optim. 44 (2005), no. 4, 1474-1528.

[108] J.A. Ball, C. Sadosky, and V. Vinnikov, Scattering systems with several evolutions and multidimensional input/state/output systems, Integral Equations Operator Theory $\mathbf{5 2}$ (2005), no. 3, 323-393.

[109] J.A. Ball and A.J. Sasane, Equivalence of a behavioral distance and the gap metric, Systems Control Lett. 55 (2006), no. 3, 214-222.

[110] J.A. Ball and O.J. Staffans, Conservative state-space realizations of dissipative system behaviors, Integral Equations Operator Theory 54 (2006), no. 2, 151-213.

[111] J.A. Ball, G. Groenewald, and T. Malakorn, Bounded real lemma for structured noncommutative multidimensional linear systems and robust control, Multidimens. Syst. Signal Process. 17 (2006), no. 2-3, 119-150.

[112] J.A. Ball and M.W. Raney, Discrete-time dichotomous well-posed linear systems and generalized Schur-Nevanlinna-Pick interpolation, Complex Anal. Oper. Theory 1 (2007), no. 1, 1-54. 
[113] J.A. Ball, V. Bolotnikov, and Q. Fang, Transfer-function realization for multipliers of the Arveson space, J. Math. Anal. Appl. 333 (2007), no. 1, 68-92.

[114] J.A. Ball and V. Bolotnikov, Interpolation in the noncommutative Schur-Agler class, J. Operator Theory 58 (2007), no. 1, 83-126.

[115] J.A. Ball, V. Bolotnikov, and Q. Fang, Multivariable backward-shift-invariant subspaces and observability operators, Multidimens. Syst. Signal Process. 18 (2007), no. 4, 191-248.

[116] J.A. Ball, P.T. Carroll, and Y. Uetake, Lax-Phillips scattering theory and wellposed linear systems: a coordinate-free approach, Math. Control Signals Systems 20 (2008), no. 1, 37-79.

[117] J.A. Ball, V. Bolotnikov, and Q. Fang, Schur-class multipliers on the Arveson space: de Branges-Rovnyak reproducing kernel spaces and commutative transfer-function realizations, J. Math. Anal. Appl. 341 (2008), no. 1, 519-539.

[118] J.A. Ball and D.S. Kaliuzhnyi-Verbovetskyi, Conservative dilations of dissipative multidimensional systems: the commutative and non-commutative settings, Multidimens. Syst. Signal Process. 19 (2008), no. 1, 79-122.

[119] J.A. Ball and V. Bolotnikov, Interpolation problems for Schur multipliers on the Drury-Arveson space: from Nevanlinna-Pick to abstract interpolation problem, Integral Equations Operator Theory 62 (2008), no. 3, 301-349.

[120] J.A. Ball, Q. Fang, G.J. Groenewald, and S. ter Horst, Equivalence of robust stabilization and robust performance via feedback, Math. Control Signals Systems 21 (2009), no. 1, 51-68.

[121] J.A. Ball, V. Bolotnikov, and S. ter Horst, A constrained Nevanlinna-Pick interpolation problem for matrix-valued functions, Indiana Univ. Math. J. 59 (2010), no. 1, $15-51$.

[122] J.A. Ball, V. Bolotnikov, and S. ter Horst, Interpolation in de Branges-Rovnyak spaces, Proc. Amer. Math. Soc. 139 (2011), no. 2, 609-618.

[123] J.A. Ball, Multidimensional circuit synthesis and multivariable dilation theory, Multidimens. Syst. Signal Process. 22 (2011), no. 1-3, 27-44.

[124] J.A. Ball and A. Kheifets, The inverse commutant lifting problem. I: Coordinatefree formalism, Integral Equations Operator Theory 70 (2011), no. 1, 17-62.

[125] J.A. Ball, V. Bolotnikov, and S. ter Horst, Abstract interpolation in vector-valued de Branges-Rovnyak spaces, Integral Equations Operator Theory 70 (2011), no. 2, $227-263$.

[126] J.A. Ball, G.M. Boquet, and V. Vinnikov, A behavioral interpretation of Livšic systems, Multidimens. Syst. Signal Process. 23 (2012), no. 1-2, 17-48.

[127] J.A. Ball and A.J. Sasane, Extension of the $\nu$-metric, Complex Anal. Oper. Theory 6 (2012), no. 1, 65-89.

[128] J.A. Ball and V. Bolotnikov, Canonical transfer-function realization for Schur multipliers on the Drury-Arveson space and models for commuting row contractions, Indiana Univ. Math. J. 61 (2012), no. 2, 665-716.

[129] J.A. Ball and M.D. Guerra Huamán, Test functions, Schur-Agler classes and transfer-function realizations: the matrix-valued setting, Complex Anal. Oper. Theory 7 (2013), no. 3, 529-575. 
[130] J.A. Ball and V. Bolotnikov, Weighted Bergman spaces: shift-invariant subspaces and input/state/output linear systems, Integral Equations Operator Theory $\mathbf{7 6}$ (2013), no. 3, 301-356.

[131] J.A. Ball and A. Kheifets, The inverse commutant lifting problem. II: Hellinger functional-model spaces, Complex Anal. Oper. Theory 7 (2013), no. 4, 873-907.

[132] J.A. Ball and V. Bolotnikov, A Beurling type theorem in weighted Bergman spaces, C. R. Math. Acad. Sci. Paris 351 (2013), no. 11-12, 433-436.

[133] J. Agler, J.A. Ball, and J.E. McCarthy, The Takagi problem on the disk and bidisk, Acta Sci. Math. (Szeged) 79 (2013), no. 1-2, 63-78.

[134] J.A. Ball and V. Bolotnikov, Weighted Hardy spaces: shift invariant and coinvariant subspaces, linear systems and operator model theory, Acta Sci. Math. (Szeged) 79 (2013), no. 3-4, 623-686.

[135] J.A. Ball and M.D. Guerra Huamán, Convexity analysis and the matrix-valued Schur class over finitely connected planar domains, J. Operator Theory $\mathbf{7 0}$ (2013), no. 2, 531-571.

[136] J.A. Ball and D.S. Kaliuzhnyi-Verbovetskyi, Rational Cayley inner Herglotz-Agler functions: positive-kernel decompositions and transfer-function realizations, Linear Algebra Appl. 456 (2014), 138-156.

[137] J.A. Ball, M. Kurula, O.J. Staffans, and H. Zwart, De Branges-Rovnyak realizations of operator-valued Schur functions on the complex right half-plane, Complex Anal. Oper. Theory 9 (2015), no. 4, 723-792.

[138] J.A. Ball, D.S. Kaliuzhnyi-Verbovetskyi, C. Sadosky, and V. Vinnikov, Scattering systems with several evolutions and formal reproducing kernel Hilbert spaces, Complex Anal. Oper. Theory 9 (2015), no. 4, 827-931.

[139] J.A. Ball and D.S. Kaliuzhnyi-Verbovetskyi, Schur-Agler and Herglotz-Agler classes of functions: positive-kernel decompositions and transfer-function realizations, Adv. Math. 280 (2015), 121-187.

[140] J.A. Ball, G. Groenewald, and S. ter Horst, Bounded real lemma and structured singular value versus diagonal scaling: the free noncommutative setting, Multidimens. Syst. Signal Process. 27 (2016), no. 1, 217-254.

[141] J.A. Ball, G. Marx, and V. Vinnikov, Noncommutative reproducing kernel Hilbert spaces, J. Funct. Anal. 271 (2016), no. 7, 1844-1920.

[142] J.A. Ball, K.F. Clancey, and V. Vinnikov, Meromorphic matrix trivializations of factors of automorphy over a Riemann surface, Oper. Matrices 10 (2016), no. 4, 785-828.

[143] J.A. Ball and V. Bolotnikov, Contractive multipliers from Hardy space to weighted Hardy space, Proc. Amer. Math. Soc. 145 (2017), no. 6, 2411-2425.

[144] J.A. Ball, M. Kurula, and O.J. Staffans, A conservative de Branges-Rovnyak functional model for operator Schur functions on $\mathbb{C}^{+}$, Complex Anal. Oper. Theory 12 (2018), no. 4, 877-915.

[145] J.A. Ball, G.J. Groenewald, and S. ter Horst, Standard versus strict bounded real lemma with infinite-dimensional state space. I. The state-space-similarity approach, J. Operator Theory 80 (2018), no. 1, 225-253. 


\section{Papers published as book chapters}

[1] J.A. Ball, A non-Euclidean Lax-Beurling theorem with applications to matricial Nevanlinna-Pick interpolation, Toeplitz centennial (Tel Aviv, 1981), Operator Theory: Adv. Appl., vol. 4, Birkhäuser, Basel-Boston, Mass., 1982, pp. 67-84.

[2] J.A. Ball, Invariant subspace representations, unitary interpolants and factorization indices, Topics in operator theory systems and networks (Rehovot, 1983), Oper. Theory Adv. Appl., vol. 12, Birkhäuser, Basel, 1984, pp. 11-38.

[3] J.A. Ball and I. Gohberg, Classification of shift invariant subspaces of matrices with Hermitian form and completion of matrices, Operator theory and systems (Amsterdam, 1985), Oper. Theory Adv. Appl., vol. 19, Birkhäuser, Basel, 1986, pp. 23-85.

[4] J.A. Ball and A.C.M. Ran, Left versus right canonical Wiener-Hopf factorization, Constructive methods of Wiener-Hopf factorization, Oper. Theory Adv. Appl., vol. 21, Birkhäuser, Basel, 1986, pp. 9-38.

[5] J.A. Ball, C. Foiaş, J.W. Helton, and A. Tannenbaum, Nonlinear interpolation theory in $H^{\infty}$, Modelling, robustness and sensitivity reduction in control systems (Groningen, 1986), NATO Adv. Sci. Inst. Ser. F Comput. Systems Sci., vol. 34, Springer, Berlin, 1987, pp. 31-46.

[6] J.A. Ball and D.W. Luse, Sensitivity minimization as a Nevanlinna-Pick interpolation problem, Modelling, robustness and sensitivity reduction in control systems (Groningen, 1986), NATO Adv. Sci. Inst. Ser. F Comput. Systems Sci., vol. 34, Springer, Berlin, 1987, pp. 451-462.

[7] J.A. Ball, Nevanlinna-Pick interpolation: generalizations and applications, Surveys of some recent results in operator theory, Vol. I, Pitman Res. Notes Math. Ser., vol. 171, Longman Sci. Tech., Harlow, 1988, pp. 51-94.

[8] J.A. Ball, I. Gohberg, and L. Rodman, Realization and interpolation of rational matrix functions, Topics in interpolation theory of rational matrix-valued functions, Oper. Theory Adv. Appl., vol. 33, Birkhäuser, Basel, 1988, pp. 1-72.

[9] J.A. Ball, N. Cohen, and A.C.M. Ran, Inverse spectral problems for regular improper rational matrix functions, Topics in interpolation theory of rational matrix-valued functions, Oper. Theory Adv. Appl., vol. 33, Birkhäuser, Basel, 1988, pp. 123-173.

[10] J.A. Ball and J.W. Helton, Shift invariant subspaces, passivity, reproducing kernels and $H^{\infty}$-optimization, Contributions to operator theory and its applications (Mesa, AZ, 1987), Oper. Theory Adv. Appl., vol. 35, Birkhäuser, Basel, 1988, pp. 265-310.

[11] J.A. Ball and J.W. Helton, Factorization and general properties of nonlinear Toeplitz operators, The Gohberg anniversary collection, Vol. II (Calgary, AB, 1988), Oper. Theory Adv. Appl., vol. 41, Birkhäuser, Basel, 1989, pp. 25-41.

[12] J.A. Ball, I. Gohberg, and L. Rodman, Two-sided Nudel'man interpolation problems for rational matrix functions, Analysis and partial differential equations, Lecture Notes in Pure and Appl. Math., vol. 122, Dekker, New York, 1990, pp. 371-416.

[13] J.A. Ball, I. Gohberg, and L. Rodman, Sensitivity minimization and bitangential Nevanlinna-Pick interpolation in contour integral form, Signal Processing, Part II, IMA Vol. Math. Appl., vol. 23, Springer, New York, 1990, pp. 3-35. 
[14] J.A. Ball, I. Gohberg, and L. Rodman, Tangential interpolation problems for rational matrix functions, Matrix theory and applications (Phoenix, AZ, 1989), Proc. Sympos. Appl. Math., vol. 40, Amer. Math. Soc., Providence, RI, 1990, pp. 59-86.

[15] J.A. Ball, I. Gohberg, and L. Rodman, Two-sided Lagrange-Sylvester interpolation problems for rational matrix functions, Operator theory: operator algebras and applications, Part 1 (Durham, NH, 1988), Proc. Sympos. Pure Math., vol. 51, Amer. Math. Soc., Providence, RI, 1990, pp. 17-83.

[16] D. Alpay, J.A. Ball, I. Gohberg, and L. Rodman, Realization and factorization for rational matrix functions with symmetries, Extension and interpolation of linear operators and matrix functions, Oper. Theory Adv. Appl., vol. 47, Birkhäuser, Basel, 1990, pp. 1-60.

[17] J.A. Ball and M. Rakowski, Zero-pole structure of nonregular rational matrix functions, Extension and interpolation of linear operators and matrix functions, Oper. Theory Adv. Appl., vol. 47, Birkhäuser, Basel, 1990, pp. 137-193.

[18] J.A. Ball and T.R. Fanney, Closability of differential operators and real sub-Jordan operators, Topics in operator theory: Ernst D. Hellinger memorial volume, Oper. Theory Adv. Appl., vol. 48, Birkhäuser, Basel, 1990, pp. 93-156.

[19] J.A. Ball and N. Cohen, de Branges-Rovnyak operator models and systems theory: a survey, Topics in matrix and operator theory (Rotterdam, 1989), Oper. Theory Adv. Appl., vol. 50, Birkhäuser, Basel, 1991, pp. 93-136.

[20] J.A. Ball, I. Gohberg, and L. Rodman, The state space method in the study of interpolation by rational matrix functions, Mathematical system theory, Springer, Berlin, 1991, pp. 503-508.

[21] J.A. Ball, I. Gohberg, and M.A. Kaashoek, Nevanlinna-Pick interpolation for timevarying input-output maps: the discrete case, Time-variant systems and interpolation, Oper. Theory Adv. Appl., vol. 56, Birkhäuser, Basel, 1992, pp. 1-51.

[22] J.A. Ball, I. Gohberg, and M.A. Kaashoek, Nevanlinna-Pick interpolation for timevarying input-output maps: the continuous time case, Time-variant systems and interpolation, Oper. Theory Adv. Appl., vol. 56, Birkhäuser, Basel, 1992, pp. 52-89.

[23] J.A. Ball, I. Gohberg, and M.A. Kaashoek, Reduction of the abstract four block problem to a Nehari problem, Continuous and discrete Fourier transforms, extension problems and Wiener-Hopf equations, Oper. Theory Adv. Appl., vol. 58, Birkhäuser, Basel, 1992, pp. 121-141.

[24] J.A. Ball and M. Rakowski, Interpolation by rational matrix functions and stability of feedback systems: the 4-block case, Operator theory and complex analysis (Sapporo, 1991), Oper. Theory Adv. Appl., vol. 59, Birkhäuser, Basel, 1992, pp. 96-142.

[25] J.A. Ball, I. Gohberg, and M.A. Kaashoek, Bitangential interpolation for inputoutput operators of time-varying systems: the discrete time case, New aspects in interpolation and completion theories, Oper. Theory Adv. Appl., vol. 64, Birkhäuser, Basel, 1993, pp. 33-72.

[26] J.A. Ball, I. Gohberg, and L. Rodman, Two-sided tangential interpolation of real rational matrix functions, New aspects in interpolation and completion theories, Oper. Theory Adv. Appl., vol. 64, Birkhäuser, Basel, 1993, pp. 73-102. 
[27] W.T. Ross and J.A. Ball, Weak-star limits of polynomials and their derivatives, Contributions to operator theory and its applications, Oper. Theory Adv. Appl., vol. 62, Birkhäuser, Basel, 1993, pp. 165-175.

[28] J.A. Ball and J. Rosenthal, Pole placement, internal stabilization and interpolation conditions for rational matrix functions: a Grassmannian formulation, Linear algebra for control theory, IMA Vol. Math. Appl., vol. 62, Springer, New York, 1994, pp. 21-29.

[29] J.A. Ball, Conservative dynamical systems and nonlinear Livšic-Brodskiǔ nodes, Nonselfadjoint operators and related topics (Beer Sheva, 1992), Oper. Theory Adv. Appl., vol. 73, Birkhäuser, Basel, 1994, pp. 67-95.

[30] J.A. Ball, I. Gohberg, and M.A. Kaashoek, Input-output operators of J-unitary timevarying continuous time systems, Operator theory in function spaces and Banach lattices, Oper. Theory Adv. Appl., vol. 75, Birkhäuser, Basel, 1995, pp. 57-94.

[31] J.A. Ball, The nonlinear Nevanlinna-Pick interpolation problem, Topics in operator theory, operator algebras and applications (Timişoara, 1994), Rom. Acad., Bucharest, 1995, pp. 1-27.

[32] J.A. Ball, I. Gohberg, and M.A. Kaashoek, The band method and Grassmannian approach for completion and extension problems, Recent developments in operator theory and its applications (Winnipeg, MB, 1994), Oper. Theory Adv. Appl., vol. 87, Birkhäuser, Basel, 1996, pp. 17-60.

[33] J.A. Ball, Linear systems, operator model theory and scattering: multivariable generalizations, Operator theory and its applications (Winnipeg, MB, 1998), Fields Inst. Commun., vol. 25, Amer. Math. Soc., Providence, RI, 2000, pp. 151-178.

[34] J.A. Ball and N.J. Young, Problems on the realization of functions, Operator theory and its applications (Winnipeg, MB, 1998), Fields Inst. Commun., vol. 25, Amer. Math. Soc., Providence, RI, 2000, pp. 179-185.

[35] J.A. Ball and T.T. Trent, The abstract interpolation problem and commutant lifting: a coordinate-free approach, Operator theory and interpolation (Bloomington, IN, 1996), Oper. Theory Adv. Appl., vol. 115, Birkhäuser, Basel, 2000, pp. 51-83.

[36] J.A. Ball, T.T. Trent, and V. Vinnikov, Interpolation and commutant lifting for multipliers on reproducing kernel Hilbert spaces, Operator theory and analysis (Amsterdam, 1997), Oper. Theory Adv. Appl., vol. 122, Birkhäuser, Basel, 2001, pp. 89-138.

[37] J.A. Ball and V. Vinnikov, Hardy spaces on a finite bordered Riemann surface, multivariable operator model theory and Fourier analysis along a unimodular curve, Systems, approximation, singular integral operators, and related topics (Bordeaux, 2000), Oper. Theory Adv. Appl., vol. 129, Birkhäuser, Basel, 2001, pp. 37-56.

[38] J.A. Ball, L. Rodman, and I.M. Spitkovsky, Toeplitz corona problem for algebras of almost periodic functions, Toeplitz matrices and singular integral equations (Pobershau, 2001), Oper. Theory Adv. Appl., vol. 135, Birkhäuser, Basel, 2002, pp. 25-37.

[39] J.A. Ball, K.F. Clancey, and V. Vinnikov, Concrete interpolation of meromorphic matrix functions on Riemann surfaces, Interpolation theory, systems theory and related topics (Tel Aviv/Rehovot, 1999), Oper. Theory Adv. Appl., vol. 134, Birkhäuser, Basel, 2002, pp. 137-156. 
[40] J.A. Ball and V. Vinnikov, Formal reproducing kernel Hilbert spaces: the commutative and noncommutative settings, Reproducing kernel spaces and applications, Oper. Theory Adv. Appl., vol. 143, Birkhäuser, Basel, 2003, pp. 77-134.

[41] J.A. Ball and V. Vinnikov, Overdetermined multidimensional systems: state space and frequency domain methods, Mathematical systems theory in biology, communications, computation, and finance (Notre Dame, IN, 2002), IMA Vol. Math. Appl., vol. 134, Springer, New York, 2003, pp. 63-119.

[42] J.A. Ball and V. Bolotnikov, Boundary interpolation for contractive-valued functions on circular domains in $\mathbb{C}^{n}$, Current trends in operator theory and its applications, Oper. Theory Adv. Appl., vol. 149, Birkhäuser, Basel, 2004, pp. 107-132.

[43] J.A. Ball and V. Vinnikov, Functional models for representations of the Cuntz algebra, Operator theory, systems theory and scattering theory: multidimensional generalizations, Oper. Theory Adv. Appl., vol. 157, Birkhäuser, Basel, 2005, pp. 1-60.

[44] J.A. Ball, G. Groenewald, and T. Malakorn, Conservative structured noncommutative multidimensional linear systems, The state space method generalizations and applications, Oper. Theory Adv. Appl., vol. 161, Birkhäuser, Basel, 2006, pp. 179 223.

[45] J.A. Ball, V. Bolotnikov, and Q. Fang, Schur-class multipliers on the Fock space: de Branges-Rovnyak reproducing kernel spaces and transfer-function realizations, Operator theory, structured matrices, and dilations, Theta Ser. Adv. Math., vol. 7, Theta, Bucharest, 2007, pp. 85-114.

[46] J.A. Ball, A. Biswas, Q. Fang, and S. ter Horst, Multivariable generalizations of the Schur class: positive kernel characterization and transfer function realization, Recent advances in operator theory and applications, Oper. Theory Adv. Appl., vol. 187, Birkhäuser, Basel, 2009, pp. 17-79.

[47] J.A. Ball and V. Bolotnikov, Canonical de Branges-Rovnyak model transfer-function realization for multivariable Schur-class functions, Hilbert spaces of analytic functions, CRM Proc. Lecture Notes, vol. 51, Amer. Math. Soc., Providence, RI, 2010, pp. 1-39.

[48] J.A. Ball and S. ter Horst, Multivariable operator-valued Nevanlinna-Pick interpolation: a survey, Operator algebras, operator theory and applications, Oper. Theory Adv. Appl., vol. 195, Birkhäuser Verlag, Basel, 2010, pp. 1-72.

[49] J.A. Ball and S. ter Horst, Robust control, multidimensional systems and multivariable Nevanlinna-Pick interpolation, Topics in operator theory. Volume 2. Systems and mathematical physics, Oper. Theory Adv. Appl., vol. 203, Birkhäuser Verlag, Basel, 2010, pp. 13-88.

[50] J.A. Ball and V. Bolotnikov, Canonical transfer-function realization for Schur-Aglerclass functions of the polydisk, A panorama of modern operator theory and related topics, Oper. Theory Adv. Appl., vol. 218, Birkhäuser/Springer Basel AG, Basel, 2012, pp. 75-122.

[51] J.A. Ball and V. Bolotnikov, Canonical transfer-function realization for Schur-Aglerclass functions on domains with matrix polynomial defining function in $\mathbb{C}^{n}$, Recent progress in operator theory and its applications, Oper. Theory Adv. Appl., vol. 220, Birkhäuser/Springer Basel AG, Basel, 2012, pp. 23-55. 
[52] J.A. Ball and A.J. Sasane, Extension of the $\nu$-metric: the $H^{\infty}$ case, Spectral theory, mathematical system theory, evolution equations, differential and difference equations, Oper. Theory Adv. Appl., vol. 221, Birkhäuser/Springer Basel AG, Basel, 2012, pp. 121-130.

[53] J.A. Ball and Q. Fang, Nevanlinna-Pick interpolation via graph spaces and Kreĭnspace geometry: a survey, Mathematical methods in systems, optimization, and control, Oper. Theory Adv. Appl., vol. 222, Birkhäuser/Springer Basel AG, Basel, 2012, pp. $43-71$.

[54] J.A. Ball and V. Bolotnikov, Interpolation in sub-Bergman spaces, Advances in structured operator theory and related areas, Oper. Theory Adv. Appl., vol. 237, Birkhäuser/Springer, Basel, 2013, pp. 17-39.

[55] J.A. Ball and V. Bolotnikov, De Branges-Rovnyak spaces: Basics and theory, Operator Theory, Springer on-line reference book, Springer, Basel, 2015, pp. 631-680.

[56] J.A. Ball and V. Bolotnikov, De Branges-Rovnyak spaces and norm-constrained interpolation, Operator Theory, Springer on-line reference book, Springer, Basel, 2015, pp. 681-720.

[57] J.A. Ball and V. Bolotnikov, On the expansive property of inner functions in weighted Hardy spaces, Complex analysis and dynamical systems VI. Part 2, Contemp. Math., vol. 667, Amer. Math. Soc., Providence, RI, 2016, pp. 47-61.

[58] J.A. Ball and V. Bolotnikov, The bitangential matrix Nevanlinna-Pick interpolation problem revisited, Indefinite inner product spaces, Schur analysis, and differential equations, Oper. Theory Adv. Appl., vol. 263, Birkhäuser/Springer, Cham, 2018, pp. 107-161.

[59] J.A. Ball, G. Marx, and V. Vinnikov, Interpolation and transfer-function realization for the noncommutative Schur-Agler class, Operator theory in different settings and related applications, Oper. Theory Adv. Appl., vol. 262, Birkhäuser/Springer, Cham, 2018, pp. 23-116.

[60] J.A. Ball, G.J. Groenewald, and S. ter Horst, Standard versus strict bounded real lemma with infinite-dimensional state space II: The storage function approach, The diversity and beauty of applied operator theory, Oper. Theory Adv. Appl., vol. 268, Birkhäuser/Springer, Cham, 2018, pp. 1-50.

\section{Conference proceedings papers}

[1] J.A. Ball and J.W. Helton, Interpolation with outer functions and gain equalization in amplifiers, Mathematical theory of networks and systems (Delft, 1979), Western Periodicals Co., 1979, pp. 41-49.

[2] J.A. Ball, Amplifier design, signed spectral factorization and signed interpolation, Mathematical theory of networks and systems (Santa Monica, 1981), Western Periodicals Co., 1981, pp. 1-3.

[3] J.A. Ball and J.W. Helton, Linear fractional parameterizations of matrix function spaces and a new proof of the Youla-Jabr-Bongiorno parameterization for stabilizing compensators, Mathematical theory of networks and systems (Beer Sheva, 1983), Lect. Notes Control Inf. Sci., vol. 58, Springer, London, 1984, pp. 16-23. 
[4] J.A. Ball and A.C.M. Ran, Hankel norm approximation of a rational matrix function in terms of its realization, Modelling, identification and robust control (Stockholm, 1985), North-Holland, Amsterdam, 1986, pp. 285-296.

[5] J.A. Ball, The bitangential Nevanlinna-Pick interpolation problem: a geometric approach, Proc. 26th IEEE Conference on Decision and Control (Los Angeles, 1987), IEEE, New York, 1987, pp. 1342-1343.

[6] J.A. Ball and J.W. Helton, Well-posedness of nonlinear causal feedback systems, Proc. 26th IEEE Conf. on Decision and Control (Los Angeles, 1987), IEEE, New York, 1987, pp. 152-154.

[7] J.A. Ball and J.W. Helton, Sensitivity bandwidth optimization for nonlinear feedback systems, Analysis and Control of Nonlinear Systems, North Holland, Amsterdam, 1988, pp. 123-129.

[8] J.A. Ball, Interpolation problems for null and pole structure of nonlinear systems, Proc. 27th IEEE Conf. on Decision and Control (Austin, 1988), IEEE, New York, 1988, pp. 14-19.

[9] J.A. Ball, N. Cohen, and L. Rodman, On interpolation problems for rectangular matrix polynomials, Proc. 27th IEEE Conf. on Decision and Control (Austin, 1988), IEEE, New York, 1988, pp. 1370-1372.

[10] J.A. Ball and J.W. Helton, Factorization of nonlinear systems: toward a theory for nonlinear $H^{\infty}$-infinity control, Proc. 27th IEEE Conf. on Decision and Control (Austin, 1988), IEEE, New York, 1988, pp. 2376-2381.

[11] D.W. Luse and J.A. Ball, Frequency-scale decomposition of $H^{\infty}$-disk problems, Linear circuits, systems and signal processing: theory and application (Phoenix, AZ, 1987), North-Holland, Amsterdam, 1988, pp. 573-580.

[12] J.A. Ball and I. Gohberg, Cascade decompositions of linear systems in terms of realizations, Proceedings of the 28th IEEE Conference on Decision and Control, Vol. 1-3 (Tampa, FL, 1989), IEEE, New York, 1989, pp. 2-10.

[13] J.A. Ball and J.W. Helton, $H^{\infty}$ control for nonlinear plants: connections with differential games, Proceedings of the 28th IEEE Conference on Decision and Control, Vol. 1-3 (Tampa, FL, 1989), IEEE, New York, 1989, pp. 956-962.

[14] J.A. Ball and J.W. Helton, Nonlinear $H^{\infty}$ control theory: a literature survey, Robust control of linear systems and nonlinear control (Amsterdam, 1989), Progr. Systems Control Theory, vol. 4, Birkhäuser Boston, Boston, MA, 1990, pp. 1-12.

[15] J.A. Ball and L.E. Carpenter, Realizations of products and Wiener-Hopf factors for a class of matrix functions analytic in a strip, Signal processing, scattering and operator theory, and numerical methods (Amsterdam, 1989), Progr. Systems Control Theory, vol. 5, Birkhäuser Boston, Boston, MA, 1990, pp. 291-300.

[16] J.A. Ball and M. Rakowski, Transfer functions with a given local zero-pole structure, New trends in systems theory (Genoa, 1990), Progr. Systems Control Theory, vol. 7, Birkhäuser Boston, Boston, MA, 1991, pp. 81-88.

[17] J.A. Ball, I. Gohberg, and L. Rodman, Nehari interpolation problem for rational matrix functions: the generic case, $H_{\infty}$-control theory (Como, 1990), Lecture Notes in Math., vol. 1496, Springer, Berlin, 1991, pp. 277-308. 
[18] J.A. Ball, I. Gohberg, and L. Rodman, Interpolation problems for rational matrix functions and systems theory, Recent advances in mathematical theory of systems, control, networks and signal processing, I (Kobe, 1991), Mita, Tokyo, 1992, pp. 3-12.

[19] J.A. Ball, J.W. Helton, and M. Verma, A J-inner-outer factorization principle for the $H^{\infty}$ control problem, Recent advances in mathematical theory of systems, control, networks and signal processing, I (Kobe, 1991), Mita, Tokyo, 1992, pp. 31-36.

[20] J.A. Ball, I. Gohberg, and M.A. Kaashoek, Time-varying systems: Nevanlinna-Pick interpolation and sensitivity minimization, Recent advances in mathematical theory of systems, control, networks and signal processing, I (Kobe, 1991), Mita, Tokyo, 1992, pp. 53-58.

[21] J.A. Ball and M. Rakowski, An application of valuation theory to the construction of rectangular matrix functions, Directions in matrix theory (Auburn, 1990), Linear Algebra Appl., Vol. 162/164, 1992, pp. 730-735.

[22] J.A. Ball, J.W. Helton, and M.L. Walker, Nonlinear $H^{\infty}$ control and the bounded real lemma, Proc. 31st Conf. on Decision and Control (Tuscon, 1992), pp. 1045-1049.

[23] J.A. Ball, I. Gohberg, and M.A. Kaashoek, The time-varying two-sided Nudel'man interpolation problem and its solution, Challenges of a generalized system theory (Amsterdam, 1992), Konink. Nederl. Akad. Wetensch. Verh. Afd. Natuurk. Eerste Reeks, vol. 40, North-Holland, Amsterdam, 1993, pp. 45-58.

[24] J.A. Ball, I. Gohberg, and M.A. Kaashoek, $H_{\infty}$-control and interpolation for timevarying systems, Systems and networks: mathematical theory and applications, Vol. I (Regensburg, 1993), Math. Res., vol. 77, Akademie-Verlag, Berlin, 1994, pp. 33-48.

[25] J.A. Ball and M. Verma, A factorization principle for stabilization of nonlinear, timevarying plants, Systems and networks: mathematical theory and applications, Vol. II (Regensburg, 1993), Math. Res., vol. 79, Akademie-Verlag, Berlin, 1994, pp. 53-56.

[26] A.J. van der Schaft and J.A. Ball, Inner-outer factorization of nonlinear state space systems, Systems and networks: mathematical theory and applications, Vol. II (Regensburg, 1993), Math. Res., vol. 79, Akademie-Verlag, Berlin, 1994, pp. 529-532.

[27] A.J. van der Schaft and J.A. Ball, Nonlinear inner-outer factorization, Proc. 33rd IEEE Conference on Decision and Control (Orlando, 1994), IEEE, New York, 1994, pp. 2549-2552.

[28] J.A. Ball, Nevanlinna-Pick interpolation and robust control for time-varying systems, Smart Structures and Materials 1996: Mathematics and Control in Smart Structures (San Diego, 1996), Proceedings of SPIE - The International Society for Optical Engineering, vol. 2715, International Society for Optics and Photonics, 1996, pp. $76-86$.

[29] J.A. Ball, M.V. Day, P. Kachroo, and T. Yu, An isolated traffic intersection feedback control using $H_{\infty}$, Intelligent Transportation System (Boston, 1997), Proc. IEEE Conference on Intelligent Transportation Systems, IEEE, New York, 1997, pp. 942947.

[30] N. Schlegel, P. Kachroo, J.A. Ball, and J.S. Bay, Image processing based control for scaled automated vehicles, Intelligent Transportation System (Boston, 1997), Proc. IEEE Conference on Intelligent Transportation Systems, IEEE, New York, 1997, pp. 1022-1027. 
[31] J.A. Ball, M.V. Day, P. Kachroo, and T. Yu, Robust control for signalized intersections, Intelligent Transportation Systems (Pittsburgh, 1997), Proceedings of SPIE The International Society for Optical Engineering, vol. 3207, International Society for Optics and Photonics, 1998, pp. 164-171.

[32] J.A. Ball and V. Vinnikov, Noncommutative linear system theory, formal power series in noncommuting indeterminates and applications, Multidimensional (nD) Systems (Wuppertal, 2005), Proc. nDS 2005, IEEE, New York, 2005, pp. 36-42.

[33] J.A. Ball, Dissipative noncommutative multidimensional linear systems and robust control theory, Multidimensional (nD) Systems (Aveiro, 2007), Proc. nDS 2007, IEEE, New York, 2007, pp. 123-129.

[34] J.A. Ball, Q. Fang, G.J. Groenewald, and S. ter Horst, Reduction of robust performance via feedback to robust stabilization, Proceedings of the 18th International Symposium on Mathematical Theory of Networks and Systems (Blacksburg, 2008).

[35] J.A. Ball and G.M. Boquet, Livšic realization of 2 D-behaviors with degree one autonomy, Multidimensional (nD) Systems (Thessaloniki, 2009), Proc. nDS 2009, IEEE, New York, 2009.

[36] J.A. Ball and S. ter Horst, $A W^{*}$-correspondence approach to multi-dimensional linear dissipative systems, Multidimensional (nD) Systems (Thessaloniki, 2009), Proc. nDS 2009, IEEE, New York, 2009.

[37] J.A. Ball and G.M. Boquet, Controllability of autonomous behaviors and Livšic overdetermined systems as $2 D$ behaviors with pure autonomy degree one, Proceedings of the 19th International Symposium on Mathematical Theory of Networks and Systems (Budapest, 2010).

[38] J.A. Ball and S. ter Horst, Robust Control, Multidimensional Systems and Multivariable Function Theory: Commutative and Noncommutative Settings, Proceedings of the 19th International Symposium on Mathematical Theory of Networks and Systems (Budapest, 2010).

[39] J.A. Ball and T. Malakorn, Structured noncommutative multidimensional linear systems and scale-recursive modeling, Proceedings of the 19th International Symposium on Mathematical Theory of Networks and Systems (Budapest, 2010).

[40] J.A. Ball and V. Bolotnikov, System theory techniques for function theory on Bergman spaces, Proceedings of the 21st International Symposium on Mathematical Theory of Networks and Systems (Groningen, 2014).

[41] J.A. Ball, G.J. Groenewald, and S. ter Horst, Structured Singular Values versus Diagonal Scaling: the Noncommutative Setting, Proceedings of the 21st International Symposium on Mathematical Theory of Networks and Systems (Groningen, 2014).

[42] J.A. Ball, G.J. Groenewald, and S. ter Horst, Bounded Real Lemma: the infinite dimensional case, Proceedings of the 22nd International Symposium on Mathematical Theory of Networks and Systems (Minneapolis, 2016).

\section{Edited books}

[1] S. Saitoh, D. Alpay, J.A. Ball, and T. Ohsawa (eds.), Reproducing kernels and their applications, International Society for Analysis, Applications and Computation, vol. 3, Kluwer Academic Publishers, Dordrecht, 1999. 
[2] J.A. Ball, J.W. Helton, M. Klaus, and L. Rodman (eds.), Current trends in operator theory and its applications, Operator Theory: Advances and Applications, vol. 149, Birkhäuser Verlag, Basel, 2004.

[3] J.A. Ball, Y. Eidelman, J.W. Helton, V. Olshevsky, and J. Rovnyak (eds.), Recent advances in matrix and operator theory, Operator Theory: Advances and Applications, vol. 179, Birkhäuser Verlag, Basel, 2008.

[4] J.A. Ball, V. Bolotnikov, J.W. Helton, L. Rodman, and I.M. Spitkovsky (eds.), Topics in operator theory. Volume 1. Operators, matrices and analytic functions, Operator Theory: Advances and Applications, vol. 202, Birkhäuser Verlag, Basel, 2010. A tribute to Israel Gohberg on the occasion of his 80th birthday.

[5] J.A. Ball, V. Bolotnikov, J.W. Helton, L. Rodman, and I.M. Spitkovsky (eds.), Topics in operator theory. Volume 2. Systems and mathematical physics, Operator Theory: Advances and Applications, vol. 203, Birkhäuser Verlag, Basel, 2010. A tribute to Israel Gohberg on the occasion of his 80th birthday.

[6] J.A. Ball, R.E. Curto, S.M. Grudsky, J.W. Helton, R. Quiroga-Barranco, and N.L. Vasilevski (eds.), Recent progress in operator theory and its applications, Operator Theory: Advances and Applications, vol. 220, Birkhäuser/Springer Basel AG, Basel, 2012 .

[7] W. Arendt, J.A. Ball, J. Behrndt, K.-H. Förster, V. Mehrmann, and C. Trunk (eds.), Spectral theory, mathematical system theory, evolution equations, differential and difference equations, Operator Theory: Advances and Applications, vol. 221, Birkhäuser/Springer Basel AG, Basel, 2012.

[8] J.A. Ball, M.A. Dritschel, A.F.M. ter Elst, P. Portal, and D. Potapov (eds.), Operator theory in harmonic and non-commutative analysis, Operator Theory: Advances and Applications, vol. 240, Birkhäuser/Springer, Cham, 2014. 


\section{Ph.D. Students of J.A. Ball}

1. Thomas R. Fanney, Closability of Differential Operators and Subjordan Operators, May 1989.

2. Marek Rakowski, Zero-pole Interpolation of Nonregular Rational Matrix Functions, December 1989.

3. Jeongook Kang [Kim], Interpolation by Rational Matrix Functions with Minimal McMillan Degree, December 1990.

4. Lonnie Carpenter, Cascade Analysis and Synthesis of Transfer Functions of Infinite Dimensional Linear Systems, May 1992.

5. Tusheng Yu, On-line Traffic Signalization using Robust Feedback Control, January 1998.

6. Jerawan Chudoung, Robust Control for Hybrid Systems with Applications to Network Traffic Problems, May 2000.

7. Tanit Malakorn, Multidimensional Linear Systems and Robust Control, May 2003.

8. Pushkin Kachroo, Optimal and Feedback Control for Hyperbolic Conservation Laws, June 2007.

9. Quanlei Fang, Multivariable Interpolation Problems, July 2008.

10. Grant M. Boquet, Geometric Properties of Over-Determined Systems of Linear Partial Difference Equations, February 2010.

11. Daniel Sutton, Structure of Invariant Subspaces for Left Invertible Operators on Hilbert Space, July 2010.

12. Moisés D. Guerra Huamán, Schur class of finitely connected planar domains: the test-function approach, April 2011.

13. Austin J. Amaya, Beurling-Lax Representations of Shift-Invariant Spaces, Zero-Pole Data Interpolation, and Dichotomous Transfer Function Realizations: Half-Plane/Continuous-Time Versions, April 2012.

14. Gregory Marx, Noncommutative Kernels, June 2017. 


\title{
Personal Reminiscences
}

\author{
Quanlei Fang, J. William Helton, Sanne ter Horst, \\ Alexander Kheifets, André C.M. Ran and James Rovnyak
}

This chapter contains personal notes from some of Joe's students and colleagues on the occasion of his seventieth birthday.

\section{Quanlei Fang}

An ancient Chinese proverb says, "One day's teacher, a whole life's father." It means that even if someone is your teacher for only a day, you should regard him like your father for the rest of your life. The word for thesis advisor in German is "Doktorvater", a father figure who will guide you through the doctorate. The idea of a father for a doctorate was about right for how I felt Professor Ball has treated me. It is also an easy role to accept, as he was about the same age as my parents.

Professor Ball is truly knowledgeable and very much respected. He is always energetic and never stops working. Sometimes he seems to be strict and I am kinda afraid of failing to meet his expectations. But I have realized that he is just trying to help out. When I said something wrong, he would make sure to point it out. I even learned the correct pronunciation of many words from him. When I need help or guidance he responds promptly, even long after I graduated from Virginia Tech. He always encourages me to attend conferences to check out recent developments in the field. I still remember that the first IWOTA I attended was in 2005, when I was about to start my third year in graduate school. The conference was held in Connecticut. Professor Ball and Professor Klaus decided to drive together from Blacksburg to Storrs where the conference was held. I remember how grateful I was since I did not know how to drive at that time and the professors shared the long driving. During my last year of graduate school, my thesis progressed slowly due to my distraction with many other things. Professor Ball was really patient and supportive at that time. 
In his free time Professor Ball enjoys singing and playing piano in the church. His wife has been very active too. I went to see their performances a few times and they were very nice. Occasionally Professor Ball and his wife Amelia would invite us (my mathematical siblings and some of his visitors at that time) to have dinner and watch football games at their home. I learned how to make sushi from Amelia. There were lots of good memories.

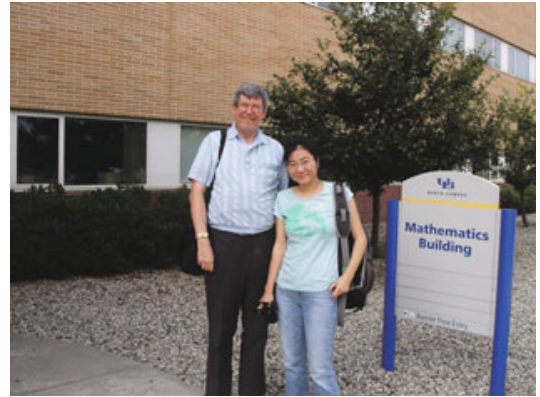

Figure 1. a) Univ. at Buffalo, 2009

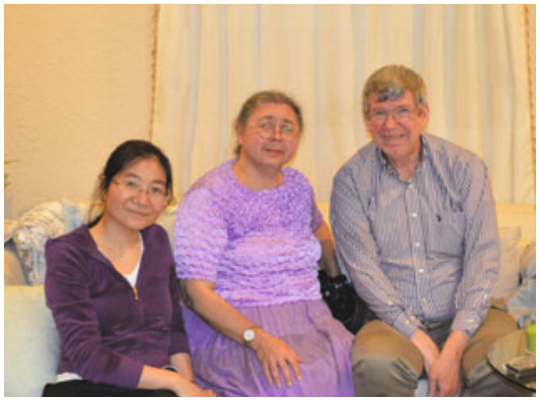

b) New York City, 2015

Professor Ball has set an example of excellence as a researcher, advisor and mentor. I feel lucky to be one of his students and I believe my other mathematical siblings feel the same. On the occasion of his 70th birthday, I just want to say: Thank you! Have a happy, healthy, wonderful birthday, and many more to come!

\section{J. William Helton}

I first heard of Joe from Tom Kriete. Tom said they had a remarkable student at UVA who, among other things, took a problem and a week later came back with an 80 page manuscript. Tom was really struck with the Joe Ball phenomenon.

Possibly the biggest branch of Operator Theory at the time was operator model theory, and Joe already was a serious expert on this. It was discovered about this time that operator model theory was very closely related to engineering systems theory, and a few of us had at a conference on this in 1973 called the Operator Theory of Networks and Systems (OTNS). So when a second OTNS was scheduled in 1975, I contacted Joe and suggested he might be interested. Joe came and probably that is where we first met (or at least talked much). There was Joe, Doug Clark and me and about 75 theoretical engineers.

Joe missed the next OTNS (which by now is called the MTNS) in 1977; it was discouraging to lose such a powerful convert to the cause.

It turned out that Joe had not lost interest; he was even shyer, at the time, than I was and later told me that he felt the conference so exalted it would be inappropriate for him to attend. 
One of the turning points in my career was getting Joe to visit San Diego for a year around 1980. Thus began a long and rewarding collaboration; every summer he would come to UCSD and also occasionally spend a quarter. This continued for about a decade and then we moved in different directions. Joe was an absolutely wonderful person to work with!

In 1981 with Joe visiting UCSD we set up the first International Workshop on Operator Theory and its Applications (IWOTA). The MTNS 1981 was set for LA and I was an organizer, so it was easy to put IWOTA as a satellite in the same hotel. Joe was a great asset in deciding who we might interest in the conference, because even then Joe had an encyclopedic knowledge of the literature. So though he did not know some of the mathematicians personally he had an opinion of their work. The conference was a big success, so much so that next summer we are expecting to have the 30th IWOTA, with Joe playing a pivotal vice presidential role on the IWOTA steering committee.

As we jump to the present, much of the work in multivariable Operator Theory depends on ideas which Joe introduced. This modern advance was introduced by Joe with Tavan Trent and brought to analytic function problems the incredibly powerful techniques of multivariable systems. Ten years later, with his student Malakorn and with Groenewald, he introduced similar techniques but for problems with operator variables. Since this is the area where I work, I am constantly feeling grateful to Joe for providing the techniques which have opened up this subject.

Joe, of course, is a backbone of our field. In addition to his work he keeps many things we depend on functioning, one of which is getting a lot of good papers refereed and published and another pertains to putting the umph in numerous conferences.

In conclusion, let us all raise a cup of coffee to Joe, in a toast to his continued bright future.

\section{Sanne ter Horst}

It must have been at one of my first international conferences, either MTNS-2004 in Louvain, Belgium, or IWOTA-2004 in Newcastle, where I first encountered Joe Ball. This was at the end of my first year as a Ph.D. student. I was working on a topic in commutant lifting theory and metric constrained interpolation, both of which Joe worked on extensively, and I had already read some of his papers. We didn't speak at that occasion, but Joe certainly made a lasting impression. Towards the end of my studies I had two ring binders full of his papers. At subsequent conferences there was more interaction, and I was very glad when Joe agreed to host me for a three months visit in the last year of my studies. One of my advisors, André Ran, who did a postdoctoral fellowship with Joe about twenty years earlier, had already prepared me: "Quite possibly he already signed you up for a colloquium." And indeed, it was during this visit that I gave my first colloquium lecture. The visit was very fruitful, I learned a lot about multidimensional 
systems as well as $C^{*}$-algebras and $C^{*}$-correspondences, and the basis for our first paper, jointly with Animikh Biswas and Quanlei Fang, was laid there. The paper was finished in the months following the visit, the last week in a 24 hour writing process, with the USA team taking one half of the day and I the other half from the Netherlands, to meet the IWOTA-2006 proceedings (OT 187) deadline. That was certainly a nice experience, and I was very happy when later that year I was awarded a two-year postdoctoral fellowship at Virginia Tech to continue working with Joe.

A few months later, everything was arranged, and I was back on a plane towards Roanoke, VA, the closest airport near Blacksburg. My new office was on the same floor and only four doors away from Joe's. As an operator theorist, Joe was a bit isolated, but he maintained good contact with some of the applied mathematicians, especially of the model reduction group, and was an active member of the department. He organized a weekly seminar on topics in operator theory and control systems, which was mostly frequented by his many graduate students, at that time Quanlei Fang, Grant Boquet, Daniel Sutton, Moisés Huamán and Austin Amaya, and there were many visitors, amongst others Animikh Biswas, Vladimir Bolotnikov, Gilbert Groenewald and Victor Vinnikov.

Working with Joe can be quite intense. My postdoc years at VT have been among the most productive in my career so far. Whenever I walked over to his office with a question, this would usually lead to a detailed discussion with many anecdotes. Joe has a phenomenally detailed memory, and he would come up with precise coordinates of various related publications, not only journal and year, but also where they were stored in his office, "Oh, I did the Math Review for that paper in 1984, so it must be in this drawer." It is maybe worth pointing out here that Joe wrote more than 350 reviews for AMS Mathematical Reviews. It must have been a few less ten years ago, but anyone who visited Joe can imagine how they were stored in his office, together with many other papers, books and notes and drafts of papers that were still in progress. I hardly ever left his office with less than four papers of reading material, some related to the question I had, some that just came up in the discussion. Lunches were usually enjoyed in downtown Blacksburg, with Joe's Diner as the preferred establishment, and the mathematics discussions did not stop during lunch. Typically, a few days after a colloquium lecture, over lunch Joe would give a complete account of the topic, who had worked on it and when, and also the operator theory involved in it, if any.

During these two years we collaborated on a variety of topics, ranging from robust control problems to the $W^{*}$-correspondence approach to transfer function realization and to interpolation problems in various function spaces, and also wrote some lengthy survey papers on multivariable interpolation and multidimensional systems. It is here were I learned to work on different projects and different topics at the same time. Many other academic activities I also did in my postdoc time at VT for the first time, I wrote my first refereeing reports and got some exposure to other editorial work (Joe was one of the editors of PAMS then), co-organized my first special session at a conference and taught my first classes. Joe was always 
helpful and patiently read through my reviews, invitation e-mails, the exams I had drafted, etc. Things I now do easily, were not as straightforward to me at that time. He is also very patient towards his students, and even small kids, as one can see from the photo below where he is teaching my youngest daughter, Lian, 18 months then, to play the piano.

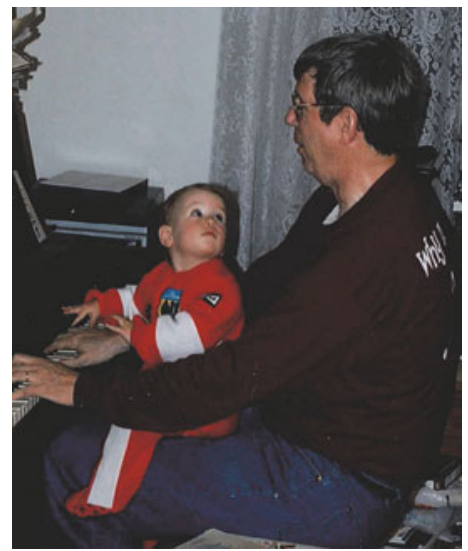

Joe learning my daughter to play the piano, 2008

Being away from my family for long periods of time wasn't easy, but Joe made very good efforts to make me feel at home. During my two years at VT, there were countless, delicious dinners at Joe's home, all carefully prepared by his wife Amelia, usually followed by an American Football match. I should admit that despite Joe's many attempts I am still lost when it comes to the rules of the game. Although Joe works long hours, often seven days a week, he also has an interesting social life. When I was there, he played the organ in church, together with Amelia he performed with the Blacksburg Master Chorale, and they attended and sometimes participated in operas at the Roanoke opera house. Many of these performances I also attended.

After my postdoc years at VT and a hiatus of a few years, we picked up our collaboration in 2012, when I had settled at North West University in South Africa. Joe visited me several times and I went back to Blacksburg in 2016 for a three weeks visit, during which I had a chance to recall the many fond memories of my time there. Joe, I want to thank you for the many things you taught me and the important role you played in my career, and especially for the great hospitality during my postdoc years at Virginia Tech.

\section{Alexander Kheifets}

I planned to meet with Joe during my first visit to the United States over the summer of 1992. I wanted to discuss with Joe the results of my recent PhD thesis, 
since there was some overlap with his works on interpolation and his "Models for non-contractions" paper, especially in the part of the adaptation of the model theory of L. de Branges and J. Rovnyak. However, we did not meet then since Joe was overseas at that time. I first met Joe a couple of years later, when I was staying as a post-doc at the Weizmann Institute of Science (Israel) and Joe was frequently visiting the Institute.

In the fall of 1995 we both participated in the Holomorphic Spaces semester at MSRI, Berkeley, CA. Although we were sharing an office for the whole semester, we did not collaborate at that time: I was working on my own paper, Joe was working with Victor Vinnikov. However, we had a lot of conversations and discussions (mathematical and non mathematical). Those discussions materialized in our later joint work.

Sometimes we worked late in our office. The window of the office looked to the sunset and we had a lot of chances to watch it. Often Joe was working there with Victor Vinnikov. Then the three of us went down the hill looking for a late dinner.

Our actual collaboration started several years later in 2001, when I was staying as a visitor at The College of William and Mary. I had some ideas on extending the Abstract Interpolation Problem approach to the general Commutant Lifting problem using linear scattering systems. I tried to discuss it with several people, but only Joe got interested in the topic and we started working on it. It was a long, really exciting and fruitful collaboration. During that time I visited Blacksburg, VA several times and Joe visited me in Lowell, MA. It is Joe's mathematical taste, understanding of the subject and persistence that allowed to complete this work.

At the beginning we included the general Commutant Lifting problem into the Abstract Interpolation problem scheme. We introduced the notion of symbol of the lift (which is a measure in the general case) and we established a one-toone correspondence between lifts and their symbols. The symbols, in turn, are solutions of the Abstract Interpolation Problem. The formula that describes the symbols of lifts of a given contraction looks rather standard (although it's precise meaning should be explained)

$$
w=s_{0}+s_{2}(I-\omega s)^{-1} \omega s_{1},
$$

where $\omega$ is a free parameter, and coefficients $s, s_{2}, s_{1}$ and $s_{0}$ are uniquely determined by the data of the problem.

However, our real objective was the inverse Commutant Lifting Problem, that consists in characterising functions $s, s_{2}, s_{1}$ and $s_{0}$ that appear in the above parametrization formulas for Commutant Lifting Problems. This type of inverse problems was studied extensively by V. Potapov for truncated Nevanlinna-Pick, Carathéodory-Fejér and Moment problems. For the infinite scalar NevanlinnaPick problem necessary conditions were obtained by R. Nevanlinna in 1929. Necessary and sufficient conditions for the Nevanlinna-Pick and some more general problems were obtained by D. Arov in the 1980s. We proved necessary and sufficient conditions for the general Commutant Lifting problem. The main difference between our "regularity" conditions and the ones obtained by D. Arov is that we 
stated ours in terms of the functional models (both coordinate-free and Hellinger space realization). Moreover, we considered the general (not only completely indeterminate case). For this general case we proved extremal properties of the coefficients $s_{2}$ and $s_{1}$, that are factoral minorants here (as opposed to equalities of the completely indeterminate case).

Numerous conversations with Joe played a significant role in improving my English. Joe was always willing to help and to answer my questions on how to say or write this or that, very often he volunteered to do that. I also got from Joe many insights on the traditions and culture of the country that was new to me. All this cannot be overestimated.

\section{André Ran}

In the early eighties a young man called Joe Ball spent a couple of months at the Vrije Universiteit in Amsterdam, and lectured there on work that he had recently done with Bill Helton. The lecture series was a very nice summary of a series of papers, and the participants of the seminar Analysis and Operator Theory learned a lot. This lecture series was my first encounter with Joe.

When finishing my $\mathrm{PhD}$ thesis became something of a certainty, and the timing of the defence became more and more certain as well, the question inevitably arose: what next? At that time, just as now, this was not something to be taken lightly. Since my first goal when starting with mathematics was to become a mathematics teacher at a high school, and since I enjoyed teaching a lot, there was certainly the option of choosing a career in that direction rather than continuing in academics. Nevertheless, after several discussions Rien Kaashoek convinced me to apply for a postdoc grant to go and visit Joe Ball for a year. And what a wonderful year it was: Joe and I wrote a series of papers, and I picked up several additional topics to work on as well. All in all, that year was decisive in my career. Joe stimulated and encouraged working on mathematics in a way that is perhaps even not deliberate. His example does the trick.

We started working on several projects: one concerning model reduction in state space terms, and one concerning inverse spectral problems in state space terms. Both projects led to multiple papers, and some of the work we did in that year formed the basis of things we did separately years later.

Joe's focus on work is exemplary, but is not appreciated by all his co-authors and friends at all times. Usually, Joe and I would have lunch at the Burger King (fries and milk to accompany our own sandwiches) and we would have a discussion on mathematics during lunch. Some time in my year there Leiba Rodman came to visit, to discuss mathematics both with Joe and with me. After one day of joining us for lunch and seeing the usual way lunch time was spent, Leiba decided he would join us for the discussion, but to have his lunch earlier: he liked to enjoy the lunch as lunch, and the discussion on mathematics for the discussion on mathematics, and never the two shall mix. 


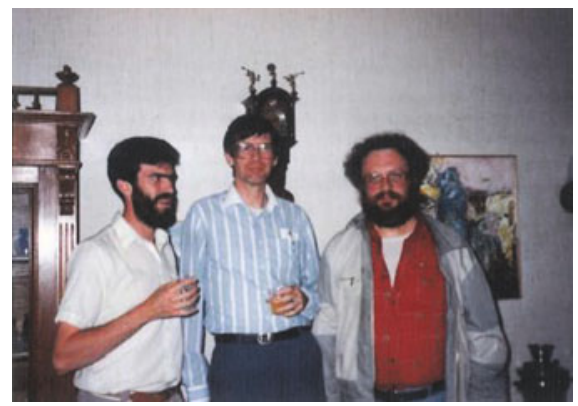

I would like to take this opportunity to thank Joe for the example he has set me, and for all the support he gave me during the early years of my career.

\section{James Rovnyak}

Joe came under the wing of Larry Shulman, a student of Louis de Branges, as an undergraduate at Georgetown University. Directly or indirectly, Larry was likely an important mentor for Joe and an influence on Joe's choice of the University of Virginia for graduate study. Larry died of leukemia, cutting a promising career tragically short.

Joe arrived at UVA in September 1969. Marvin Rosenblum's program in operator theory and classical analysis was running full steam. The faculty in operator theory around Joe's time was expanded to include Bruce Abrahamse, Jim Howland, Tom Kriete, and this writer. Graduate students were trained in a seminar that met twice a week. The seminar was one of Marvin's best ideas. A scribe was appointed and responsible to produce mimeographed notes to all participants by the next meeting. The seminar surveyed current topics in operator theory representing faculty interests and perspectives. Among the topics presented in Joe's days were canonical models, the commutant lifting theorem, and Lax-Phillips theory. Joe absorbed all and produced a masterful $\mathrm{PhD}$ thesis directed by Marvin Rosenblum. To quote my 1973 recommendation letter for Joe, the thesis is "a substantial treatise which explores the connections between 1) the model theories of de Branges-Rovnyak and Sz.-Nagy-Foias, and 2) the perturbation theories of Kato-Kuroda and de Branges-Shulman." Joe's talent was recognized early. When Joe began his teaching career, Virginia Tech was not the research powerhouse that it is today; Jimmy McShane had a high opinion of Joe and thought he should have gone to a major research university for his first job. I was present when Joe gave what was possibly his first international lecture on his work. This was at Oberwolfach, and many international mathematicians were in the audience; at the end of the lecture, Sz.-Nagy and Foiaş walked briskly up to Joe to meet this new member of the community. Joe was launched on the international scene, and the rest is history. 
Sadly, I do not recall any scandalous stories relating to Joe. I remember Joe as a serious and modest student who worked hard and produced the goods. He had an enjoyable sense of humor. I was always impressed that Joe was an organist and played at a local church in his graduate student days.

Quanlei Fang

Department of Math \& Computer Science

CUNY-BCC

Bronx, NY 10453, USA

e-mail: quanlei.fang@bcc.cuny.edu

J. William Helton

Department of Mathematics

University of California

San Diego, CA, USA

e-mail: helton@math.ucsd.edu

Sanne ter Horst

Department of Mathematics

Unit for BMI, North-West University

Potchefstroom 2531, South Africa

e-mail: Sanne.TerHorst@nwu.ac.za

Alexander Kheifets

Department of Mathematical Sciences

University of Massachusetts Lowell

One University Avenue

Lowell, MA 01854, USA

e-mail: Alexander_Kheifets@uml.edu

André C.M. Ran

Department of Mathematics

Faculty of Science, VU Amsterdam

De Boelelaan 1081a

1081 HV Amsterdam, The Netherlands

and

Unit for BMI, North-West University

Potchefstroom 2531, South Africa

e-mail: a.c.m.ran@vu.nl

James Rovnyak

University of Virginia

Department of Mathematics

P.O. Box 400137

Charlottesville, VA, 22904-4137, USA

e-mail: rovnyak@virginia.edu 